\title{
Geometric Time Delay Interferometry
}

\author{
Michele Vallisneri \\ Jet Propulsion Laboratory, California Institute of Technology, Pasadena, CA 91109
}

(Dated: Version of June 28, 2005)

\begin{abstract}
The space-based gravitational-wave observatory LISA, a NASA-ESA mission to be launched after 2012, will achieve its optimal sensitivity using Time Delay Interferometry (TDI), a LISA-specific technique needed to cancel the otherwise overwhelming laser noise in the inter-spacecraft phase measurements. The TDI observables of the Michelson and Sagnac types have been interpreted physically as the virtual measurements of a synthesized interferometer. In this paper, I present Geometric TDI, a new and intuitive approach to extend this interpretation to all TDI observables. Unlike the standard algebraic formalism, Geometric TDI provides a combinatorial algorithm to explore exhaustively the space of second-generation TDI observables (i.e., those that cancel laser noise in LISA-like interferometers with time-dependent armlengths). Using this algorithm, I survey the space of second-generation TDI observables of length (i.e., number of component phase measurements) up to 24, and I identify alternative, improved forms of the standard second-generation TDI observables. The alternative forms have improved high-frequency gravitational-wave sensitivity in realistic noise conditions (because they have fewer nulls in the gravitational-wave and noise response functions), and are less susceptible to instrumental gaps and glitches (because their component phase measurements span shorter time periods).
\end{abstract}

PACS numbers: 04.80.Nn, 07.60.Ly, 95.55.Ym

\section{INTRODUCTION}

The Laser Interferometer Space Antenna (LISA) is a joint NASA-ESA deep-space mission to be launched after 2012 , aimed at detecting and studying gravitational waves (GWs) with frequencies between $10^{-5}$ and $10^{-1} \mathrm{~Hz}$ [1]. LISA will provide access to GW sources that are outside the reach of ground-based interferometric GW detectors 2], such as the binaries of compact stellar objects in our galaxy, the mergers of massive and supermassive black holes, and the gravitational captures of compact objects by the supermassive black holes at the center of galaxies [3].

LISA consists of three widely separated spacecraft, flying around the Sun in a quasi-equilateral triangular configuration and exchanging phase-coherent laser signals. LISA relies on picometer interferometry to measure GWs as modulations in the distance between the spacecraft. The greatest challenge to achieving this measurement is the phase noise of the LISA lasers, which is larger than the GW-induced response by many orders of magnitude, and which cannot be removed by conventional phase-matching interferometry because the LISA armlengths are grossly unequal, and changing continuously. Time Delay Interferometry (TDI), developed by J. W. Armstrong, F. B. Estabrook, M. Tinto, and others [4, 5, 6, 7, 8, 9, 10, 11], is the LISA-specific technique that will be used to combine the laser-noise-laden oneway phase measurements performed between the three spacecraft ${ }^{1}$ into virtual interferometric observables where

\footnotetext{
1 A variant of the technique uses combinations of one-way and twoway phase measurements, generated by locking five of the six LISA lasers to the last one, as described by Tinto and colleagues
}

laser noise is reduced by several orders of magnitude.

TDI was initially developed using ad hoc algebraic reasoning for the case of a stationary LISA configuration with unequal but constant armlengths (first-generation TDI, see [4, 5] ). It was later modified to work also in the case of a rotating LISA constellation (modified TDI, see [8, 9, 10, 11]) and of linearly changing armlengths (second-generation TDI, see 10, 11]). First-generation and modified TDI were given a rigorous mathematical foundation in the theory of algebraic syzygies on moduli [7], providing tools to generate all possible TDI observables, and to determine which observables are optimally sensitive to GWs 13. Unfortunately, this algebraic treatment cannot be extended easily to secondgeneration TDI, which is the version that must be used in practice.

In this paper, I give a new derivation of firstgeneration, modified, and second-generation TDI, using a geometric approach that emphasizes the physical interpretation of TDI observables as synthesized interferometric measurements 4, 10, 14], extending it to all known observables. What is more, this geometric approach to TDI (in short, Geometric TDI) allows the exhaustive enumeration of all TDI observables of any length, and it leads to alternative, improved forms of the standard TDI observables, characterized by better GW sensitivity at high frequencies in realistic noise conditions, by lesser demands on the measurement system, and by reduced susceptibility to gaps and glitches.

More specifically, all TDI observables display nulls in

12]. In this paper we shall consider only the one-way formalism, but our results could be applied with superficial modifications also to the two-way variant. 


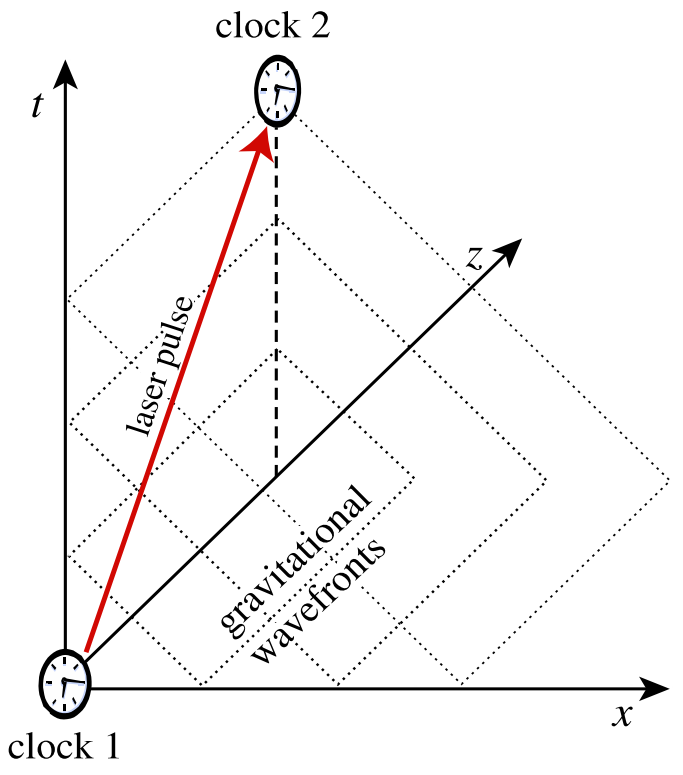

FIG. 1: In this idealization of the basic time-transport observable used with LISA, two ideal clocks travel along geodesics, with clock 1 continuously transferring its proper time to clock 2 by way of pulsed light signals. GWs are measured as the fluctuations in the time of flight between the clocks (see main text).

their noise and GW responses at frequency multiples of the inverse arm-crossing light times. ${ }^{2}$ Because these zeros occur at the same frequencies and with the same orders for noise and GWs, the ideal GW sensitivity after successful laser-noise suppression is finite, and comparable to the sensitivity at nearby frequencies. The actual sensitivity, however, is likely to be degraded, either because noise leaks into the nulls from the sides [15], or because the measurement system has insufficient dynamical range to resolve the tiny signals within the nulls. This problem is mitigated with the alternative observables, which have half as many response-function nulls as the standard forms.

In addition, because the alternative observables are, as it were, folded versions of their standard forms, they have a smaller temporal footprint: that is, they are written as sums of one-way phase measurements that span a shorter time period. This property can be advantageous in the presence of instrumental gaps or glitches, which would then contaminate a smaller portion of the data set; a reduced temporal footprint means also that a shorter continuous set of phase data needs to be collected before TDI observables can begin to be assembled.

This paper is organized as follows. Section

\footnotetext{
2 The responses are exactly null only in the limit of equal LISA armlengths. For realistic, time-evolving LISA geometries the nulls are spread into narrow dips; however, these are deep enough that the qualitative discussion to follow still applies.
}

Geometric TDI: in Sec. IIA I introduce the basic GWsensitive phase measurement; in Sec. IIIB I discuss its integration into laser-noise-canceling observables according to the Geometric TDI principle; in Secs. IIC] and IID I give a new derivation of the observables of firstgeneration, modified, and second-generation TDI, and I interpret them geometrically; in Sec. IE I show how to enumerate exhaustively all possible observables by representing them as link strings; last, in Sec. IIFI extend our formalism, developed for simplicity by considering only three independent LISA lasers, to the realistic case of six lasers. Section 【II reports on the exhaustive survey of all second-generation TDI observables consisting of up to 24 separate phase measurements: in Secs. ЩA and ЩB I discuss the alternative forms of the standard secondgeneration TDI observables, and present their practical advantages for the implementation of TDI; in Sec. IIIC I describe the previously unknown second-generation TDI observables of length 18 and more. Last, Sec. IV presents my conclusions. The appendices contain rules and proofs omitted from the main text, and explicit algebraic expression for the second-generation TDI observables of length 16 .

As customary, I set $G=c=1$ except where specified otherwise.

\section{A GEOMETRIC VIEW OF TIME-DELAY INTERFEROMETRY}

How is LISA an interferometer other than by name? The loosest dictionary definition of "interferometer" (something like "a device that combines the signals radiating from a common source, and received at different locations, or at the same location after traveling different paths") does not seem to apply to LISA, whose TDI GW observables are combinations of the phase-difference measurements between as many as six laser sources. In fact, interferometry is not needed, strictly speaking, to measure GWs, but only to remove the otherwise deafening phase noise produced by the LISA lasers. The basic principle of GW measurement employed by LISA is noninterferometric, as we can see from the idealized experimental setup of a time-transport link between two ideal clocks (see Fig. 囵).

\section{A. The basic time-transport observable}

Consider a plane GW propagating across the Minkowski background geometry, and written in the transverse-traceless gauge [16]. The wave is traveling along the $x$ direction, and has "+" polarization along the $y$ and $z$ directions. We can then write the spacetime metric as $\eta_{\mu \nu}+h_{\mu \nu}^{\mathrm{TT}}$, where

$$
h_{\mu \nu}^{\mathrm{TT}}=h_{+}(t+x)\left[\mathrm{e}_{z z}-\mathrm{e}_{y y}\right] .
$$


Consider also two ideal clocks 1 and 2, marking their proper times $t_{1}$ and $t_{2}$, and sitting at constant spatial coordinates $\vec{p}_{1}=\{0,0,0\}$ and $\vec{p}_{2}=\{0,0, L\}$ in the TT frame. In this gauge, constant-coordinate worldlines are geodesics, so the effect of the GWs is not to exert forces (as it were) on test particles, but to modulate the distance between them. By way of light signals, clock 1 is continuously sending its time $t_{1}$ to clock 2 , where $t_{1}$ is compared with the local time $t_{2}$, yielding the difference

$$
\Delta t_{12}=t_{2}(t)-t_{1}\left(t-L_{12}(t)\right)=L_{12}(t) .
$$

Here $t$ is the TT coordinate time and $L_{12}(t)$ is the time of flight between the two clocks, as experienced by the laser pulse that arrives at clock 2 at time $t$. We are assuming that the two clocks have been synchronized so that in the absence of GWs they both mark the coordinate time $t$. To first order in $h_{+}, L_{12}(t)$ is

$$
L_{12}(t)=L+\frac{1}{2} \int_{t-L}^{t} h_{+}(t) d t
$$

the $x$ coordinate dependence of the GW does not appear in Eq. (3) because the two clocks sit on the same constant- $x$ wavefronts. If the rates of the two clocks remain synchronized $\left(d t_{1} / d t=d t_{2} / d t\right)$, then the time derivative $d \Delta t_{12} / d t$ is directly proportional to difference of the GW strains at the events of pulse reception and emission,

$$
\frac{d \Delta t_{12}}{d t}=\frac{d \Delta t_{12}}{d t_{2}}=\frac{1}{2}\left[h_{+}(t)-h_{+}(t-L)\right] .
$$

This is our $G W$ observable. In the Fourier domain, the (power) response function of $d \Delta t_{12} / d t$ to GWs is $|1-\exp (-2 \pi i f L)|^{2} / 4=\sin ^{2}(\pi f L)$. Thus $d \Delta t_{12} / d t$ is insensitive to GWs of frequencies $f \ll 1 / L$ or $f \simeq k / L$ (with integer $k$ ). Expression (4) is the basic building block used to derive the LISA response to GW waves, as well as the Doppler response used in spacecraft-tracking GW searches [17], and the timing-residual response used in pulsar-timing searches [18].

To relate this idealized experimental setup to LISA, we replace the ideal clocks with the LISA lasers, and obtain proper time by dividing the lasers' phase by their frequency. Each of the three LISA spacecraft contains two optical benches oriented facing the other two spacecraft; on each bench, the appropriately named phasemeters compare the phase of the incoming lasers against the local reference laser. As written, Eq. (4) involves a comparison of laser frequencies: we choose to develop our arguments in terms of these, since it is more convenient to deal with instrumental responses that are directly proportional to the physical observable of interest (the GW strain) rather than to its time integral. Generalizing Eq. (4) to arbitrary plane-GW and spacecraft geometries, and adopting a LISA-specific language, we come to the Estabrook-Wahlquist two-pulse response [19]

$$
y_{12}(t)=\frac{1}{2} \frac{\hat{n}_{12}^{i}(t) \hat{n}_{12}^{j}(t)\left[h_{i j}^{\mathrm{TT}}\left(p_{2}^{s}(t), t\right)-h_{i j}^{\mathrm{TT}}\left(p_{1}^{s}\left(t_{\mathrm{s}}\right), t_{\mathrm{s}}\right)\right]}{1-\hat{n}_{12}^{m}(t) k_{m}}:
$$

with this indexing, Eq. (5) describes the frequencydifference measurement performed on spacecraft 2 to compare the local laser to the laser incoming from spacecraft 1 . In this equation:

- $k_{j}$ is the spatial propagation vector of the plane GW;

- $p_{1}^{s}(t)$ and $p_{2}^{s}(t)$ are the spatial TT coordinates of the two spacecraft;

- $t$ is the time of pulse reception, and therefore of measurement;

- $t_{\mathrm{s}}$ is the time of pulse emission, as determined implicitly by $\left|p_{2}^{s}(t)-p_{1}^{s}\left(t_{\mathrm{s}}\right)\right|=t-t_{\mathrm{s}}$;

- $\hat{n}_{12}^{m}(t)$ is the unit vector along the trajectory of the light pulse (labeled by the time of reception $t$ ), given by $\hat{n}_{12}^{m}(t)=\left(p_{2}^{s}(t)-p_{1}^{s}\left(t_{\mathrm{s}}\right)\right) /\left|p_{2}^{s}(t)-p_{1}^{s}\left(t_{\mathrm{s}}\right)\right|$.

Equation (5) is known as the two-pulse response because an impulsive GW is registered twice in each $y_{i j}(t)$ observable, once when it impinges on the emitting spacecraft $i$, and once, a time $L$ later, when it impinges on the receiving spacecraft $j$.

In the literature on TDI, it is customary to label the LISA arms by the index of the opposite spacecraft. We shall do so in this paper, using primed and unprimed indices to denote the oriented LISA arms (with orientation following the direction of laser transmission) according to the convention ${ }^{3}\{1,2,3\} \equiv\{3 \rightarrow 2,1 \rightarrow 3,2 \rightarrow 1\}$ and $\left\{1^{\prime}, 2^{\prime}, 3^{\prime}\right\} \equiv\{2 \rightarrow 3,3 \rightarrow 1,1 \rightarrow 2\}$. We shall then denote by $L_{l}$ the propagation time experienced by a laser pulse traveling along the oriented $\operatorname{arm} l$. We shall also find it useful, at times, to augment the $y_{i j}$ phasemeasurement notation with a middle index, corresponding to the oriented arm traversed by the laser pulse being measured. (In fact, the primed or unprimed middle index would be sufficient to identify the phase measurement completely, and we shall exploit this property in Sec. IIE when we represent TDI observables as link strings.) See Fig. 2 for an example of this convention at work.

\section{B. The Geometric TDI principle}

Unfortunately, GWs cannot be read off directly from the $y_{i j}$ measurements, because the fluctuations $C_{i}(t)$ of the laser frequencies (i.e., the laser phase noises) come into the $y_{i j}(t)$ as

$$
y_{i j}(t)=C_{i}\left(t_{\mathrm{s}}\right)-C_{j}(t)+\mathrm{GWs} ;
$$

\footnotetext{
${ }^{3}$ See the TDI Rosetta Stone, first printed in Ref. 20| and updated at www.vallis.org/tdi for a mapping between the different conventions used in the literature.
} 


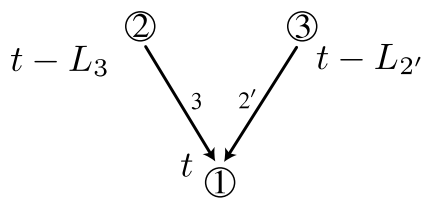

$y_{231}(t)-y_{32^{\prime} 1}(t)$

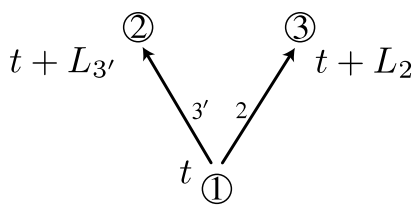

$-y_{13^{\prime 2}}\left(t+L_{3^{\prime}}\right)+y_{123}\left(t+L_{2}\right)$

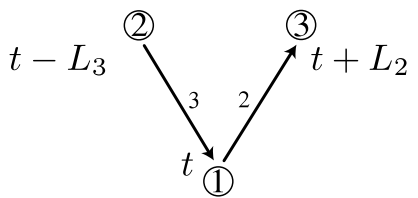

$y_{231}(t)+y_{123}\left(t+L_{2}\right)$

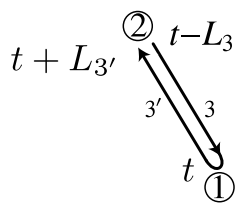

$y_{231}(t)+y_{13^{\prime 2}}\left(t+L_{3^{\prime}}\right)$
FIG. 2: These time-delayed sums and differences of two $y_{i j}$ measurements cancel laser phase noise at time $t$. In all of them, two laser pulses arrive at, or depart from, the same spacecraft at time $t$.

the LISA lasers have $C_{i}(t)$ of (single-sided, square-root) spectral density $\sim 30 \mathrm{~Hz} / \sqrt{\mathrm{Hz}}$, several orders of magnitude stronger than the weakest GWs detectable by LISA, which are at the level of the other two fundamental LISA noises (known together as secondary noises): the shot noise at the phasemeter, as determined by the power of the lasers and by the distance between the spacecraft, and the acceleration noise of the proof masses enclosed within each optical bench, which are used to reference the frequency measurements to freely falling worldlines. [Equation (6) assumes that a single laser is being used on each spacecraft; it is pedagogical to consider this simplified case first, but we shall generalize our discussion to the realistic case of six LISA lasers in Sec. [IF] ]

Canceling laser phase noise is where interferometry comes to the rescue. Look at Fig. 2 for combinations of $y_{i j}$ measurements in which two laser pulses arrive simultaneously at spacecraft 1 at time $t$, depart simultaneously from spacecraft 1 at time $t$, or arrive and depart simultaneously to and from spacecraft 1 at time $t$. We subtract the $y_{i j}$ measurements, represented graphically by arrows, when they share the same event of emission or reception (i.e., when their arrowtails or arrowheads meet), and we add them when the receiving spacecraft of one measurement is the emitting spacecraft of the other (i.e., when arrowtail follows arrowhead). In all of these combinations, the laser-frequency noise $C_{1}(t)$ generated at time $t$ on spacecraft 1 is canceled out by entering twice with opposite signs; however, GWs are not canceled (not even at time $t$ ), because they come into Eq. (5) with different $n_{i j}^{m}$-dependent projection factors. The combinations of Fig. 2 do still contain frequency noise from lasers 2 and 3 , and from times other than $t$; it is however a simple leap to cancel even those by arranging together more measurements. We then formulate a

Geometric TDI principle: to obtain a laser-noise- canceling GW observable, line up arrows (i.e., $y_{i j}$ measurements) head to head, tail to tail, or head to tail, creating a closed loop that cancels laser noise at all pulse emission and reception events. If no arrowhead or arrowtail is left unpaired, the closed loop represents a linear combination of delayed $y_{i j}$ measurements that completely cancel the three laser noises $C_{i}(t)$.

Remarkably, it is usually possible to interpret each closed-loop combination as the interferometric measurement performed by comparing the phases of laser beams that follow the paths marked by the arrows. Let us see an example. The arrows of Fig. 3 (left panel) reproduce the paths followed by light in an equal-arm Michelson interferometer; operating in analogy with Fig. 2] and attributing the time $t$ to the final common event of reception at spacecraft 1 , we write the corresponding algebraic expression

$$
y_{13^{\prime 2}}\left(t-L_{3}\right)+y_{231}(t)-y_{32^{\prime} 1}(t)-y_{123}\left(t-L_{2^{\prime}}\right) .
$$

Here the sum of two time-consecutive $y_{i j}$ observables, such as $y_{13^{\prime} 2}\left(t-L_{3}\right)$ and $y_{231}(t)$ [here $L_{3}$ is the lighttravel time between spacecraft 2 and 1 ], simulates the reflection of the laser off a mirror: in terms of laser phases, we see that the integral of this sum reproduces the total phase shift accumulated along the path $1 \rightarrow 2 \rightarrow 1$. By contrast, the head-to-head difference of two such double arrows simulates a photodetector: it reproduces the difference of the phase shifts accumulated along the two paths. All in all, Eq. (7) shows that the combination of four (one-way) $y_{i j}$ measurements can synthesize the phase-difference output of a Michelson interferometer, as emphasized by Tinto and Armstrong [4], and shown graphically by Shaddock [10] and Summers 14]. Inserting the laser noises $C_{i}(t)$ in Eq. (7), we get

$$
\begin{aligned}
{\left[C_{1}\left(t-L_{3}-L_{3^{\prime}}\right)\right.} & \left.-C_{2}\left(t-L_{3}\right)\right] \\
& +\left[C_{2}\left(t-L_{3}\right)-C_{1}(t)\right] \\
& -\left[C_{3}\left(t-L_{2^{\prime}}\right)-C_{1}(t)\right] \\
-\left[C_{1}\left(t-L_{2^{\prime}}-L_{2}\right)\right. & \left.-C_{3}\left(t-L_{2^{\prime}}\right)\right],
\end{aligned}
$$

which sums up to zero in interferometer geometries where ${ }^{4} L_{3}+L_{3^{\prime}}=L_{2^{\prime}}+L_{2}$ : our equal-arm Michelson combination is then truly laser-noise canceling. It is however sensitive to GWs, as can be seen by inserting Eq. (5) in Eq. (7).

More generally, we can set three simple rules to turn a closed arrow loop into a combination of $y_{i j}$ measurements that cancels laser noise:

1. start at any spacecraft, and write down the appropriate $y_{i j}$ for each arrow, following the loop (going

\footnotetext{
${ }^{4}$ If the interferometer is rotating, the Sagnac effect [21] introduces a distinction between the light-travel times $L_{l}$ and $L_{l^{\prime}}$ in the two directions [8, 9].
} 


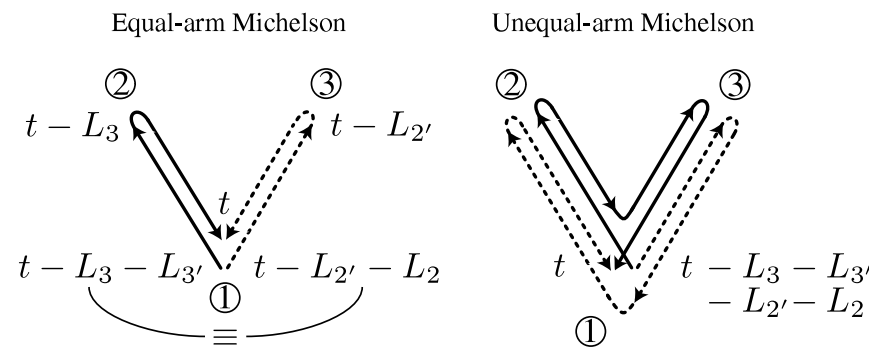

FIG. 3: Left.- The arrows of this closed loop reproduce the paths of light in a standard equal-arm Michelson interferometer, and the corresponding time-ordered sum of phase measurements [Eq. (7)] reproduces the phase-difference output of the interferometer. Right.-For unequal armlengths, laserphase-noise cancellation can be recovered by having both interfering beams travel along each arm once, building up the same light-travel time. Compare with Fig. of Ref. [10].

along or against the direction of each arrow) until all arrows are used up (if more than two heads or tails meet at any spacecraft, different visiting orders will yield different observables);

2. use a plus (minus) sign for arrows followed along (against) their direction;

3. give time arguments to the $y_{i j}$, remembering that measurements are always made at the receiving spacecraft (at the arrowhead); use the nominal time $t$ for the first $y_{i j}$, and then add (subtract) the appropriate $L_{l}$ for each arrow followed along (against) its direction.

\section{The observables of first-generation TDI}

The first laser-noise-canceling combinations for LISA were discovered using an algebraic (rather than geometric) approach, matching up delayed $y_{i j}$ measurements in such a way that all laser-noise terms would cancel. Using this procedure, Tinto, Armstrong, and Estabrook [4, 5, 6] obtained expressions for first-generation TDI observables, which cancel laser noise in static unequal-arm geometries. These observables are sums of either six or eight delayed $y_{i j}$ measurements (for short, links). See Fig. 4.

The 6 -link observables $\alpha, \beta, \gamma$ (mapped into each other by relabeling the spacecraft cyclically) use all six LISA oriented arms, and measure the phase difference accumulated by two laser beams traveling around the LISA array in clockwise and counterclockwise directions: thus, they behave much like a Sagnac interferometer, and are known as Sagnac observables. A related 6-link combination, the symmetrized Sagnac observable $\zeta$, has the useful property of being relatively insensitive to GWs in the low-frequency limit. ${ }^{5}$

The 8-link observables $X, Y, Z$ (also mapped into each other by cyclic spacecraft relabelings) use two of the LISA arms in the two directions. They are unequalarm generalizations of the Michelson observable of Eq. (7): for unequal arms, the latter would fail to cancel the laser-noise terms from the tails of the two paths, because $L_{3}+L_{3^{\prime}} \neq L_{2^{\prime}}+L_{2}$. The solution is to have both paths go through each arm once (hence the eight terms), building up the same light-travel time (see the right panel of Fig. 31). Related 8-link combinations, known as observables of the $U, P$, and $E$ type, use different sets of four oriented arms out of six, and have GW sensitivity comparable to the Michelson combinations [5, [6].

Prior to my work, it was unclear whether the $P$-type and $E$-type observables could be interpreted as synthesized interferometric observables. ${ }^{6}$ In Fig. 4 we show that this is possible if we identify four distinct laser beams, paired in alternative ways to cancel laser noise at the path tails (dots) and path heads (ending arrows). The two path origins are not simultaneous, and neither are the two path endings.

The symmetrized Sagnac observable $\zeta$, which also defies explanation as a two-beam synthesized interferometer, can be interpreted as a six-beam interferometer, whereby two different pairings explain the cancellation of laser noise at emission (dots) and reception (arrows). Yet another pairing, shown by the thin diagonal lines in Fig. (4) explains why $\zeta$ is relatively insensitive to GWs at low frequencies: in the limit of equal arms, each pair of parallel arrows represents the difference of two symmetric measurements $y_{i j}(t)$ and $y_{j i}(t)$ that share the same times of pulse emission and reception. Taylor-expanding the $h_{i j}^{\mathrm{TT}}(\ldots ; t)$ terms of Eq. (5) around $t$ and around either $p_{1}^{l}$ or $p_{2}^{l}$, we find that $y_{i j}(t)-y_{j i}(t) \propto L^{3} h_{i j}^{\prime \prime \prime}$. By contrast, the differences of head-to-tail double arrows that appear in $X$ sum up to $L^{2} h_{i j}^{\prime \prime}$. Considering monochromatic GWs of frequency $f_{\mathrm{GW}}$, we see that the GW response is smaller for $\zeta$ than for $X$ by a factor $2 \pi f_{\mathrm{GW}} L$ $\left(\simeq 0.1\right.$ for $f_{\mathrm{GW}}=10^{-3} \mathrm{~Hz}, \simeq 0.01$ for $\left.10^{-4} \mathrm{~Hz}\right)$. Since the response to the LISA secondary noises is approximately the same for $\zeta$ and $X$ [as can be seen using Eqs. [201), discussed below], $\zeta$ turns out to be relatively insensitive to GW.

\footnotetext{
${ }^{5}$ Most interestingly, a GW-insensitive observable allows the observational distinction of a stochastic GW background from instrumental noise 22].

6 The interpretation of $U$ as a synthesized observable was already clear to F. B. Estabrook (unpublished note).

7 For unequal interferometer arms, $\zeta \propto L(\Delta L) h_{i j}^{\prime \prime}$, so the ratio between the $\zeta$ and $X$ responses is $\sim \Delta L / L \sim 0.01$.
} 


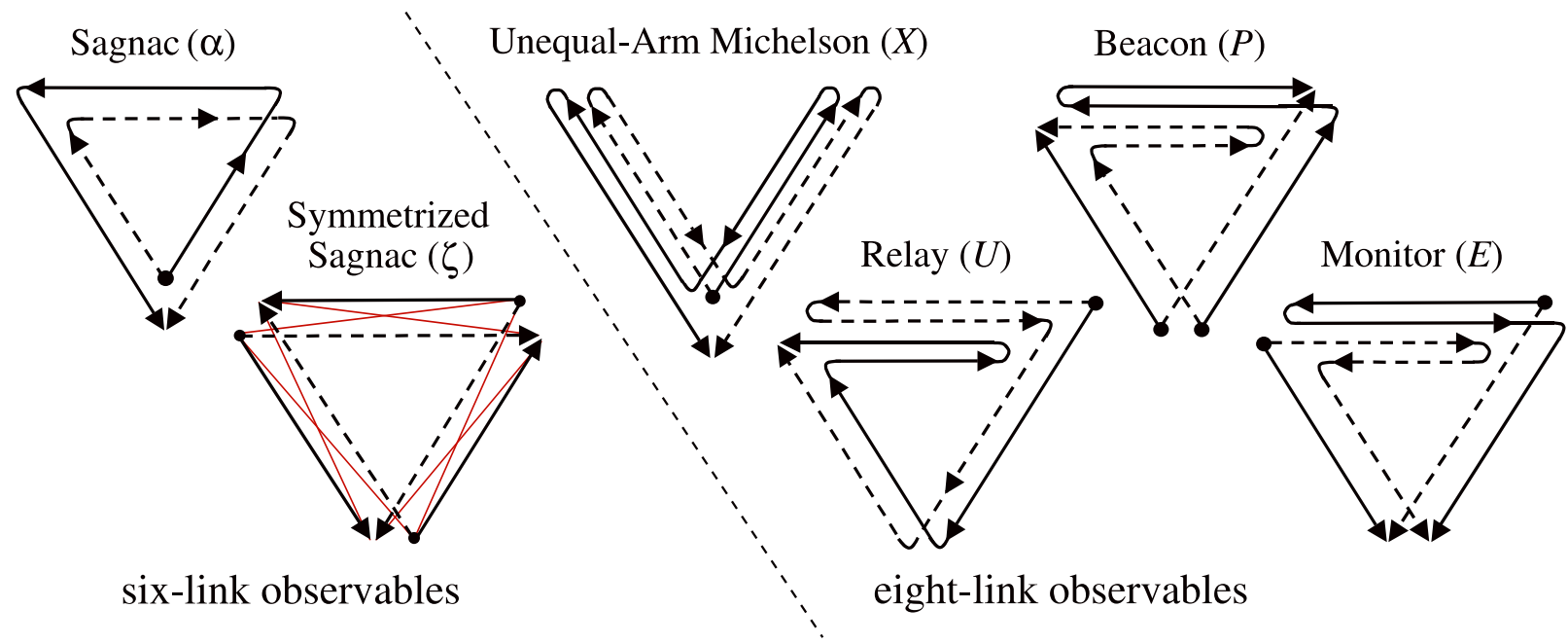

FIG. 4: Mapping first-generation TDI observables into closed arrow loops. The Sagnac $(\alpha)$, unequal-arm Michelson $(X)$, and Relay $(U)$ observables of first-generation TDI have a straightforward interpretation as synthesized two-beam interferometers. More interestingly, the Beacon $(P)$ and Monitor $(E)$ observables can be seen as four-beam interferometers, with the four beams combining into different pairs at the events of initial emission and final reception. To interpret the symmetrized Sagnac $(\zeta)$ observables as six-beam interferometers, three different beam pairings must be invoked to explain the cancellation of laser noise at emission and reception, and the relative insensitivity of $\zeta$ to GWs (see main text).

\section{The observables of second-generation TDI}

This interpretation of TDI observables as $2 N$-beam synthesized interferometers is intriguing, but also troubling, since it casts a suspicion of arbitrariness on the selection of a standard set of observables, and it complicates exploring the space of all possible combinations. Fortunately, the application of the tools of modern algebra to TDI showed that all first-generation observables can be obtained as algebraic combinations of four generators [7]. This approach was extended 7] to modified TDI observables [8, 9, 10, 11], which cancel laser noise in rotating LISA geometries, where the Sagnac effect 21] introduces a distinction between light-travel times in the two directions. (The Michelson-, U-, P-, and E-type observables of first-generation TDI are bona fide modified TDI observables, if written with the correct primed and unprimed delay indices; by contrast, the Sagnac observables of modified TDI are different, and twice as long as those of first-generation TDI.)

However, the algebraic approach cannot be extended easily to the observables of second generation TDI, which cancel laser noise in LISA geometries with timedependent armlengths. ${ }^{8}$ As pointed out by Cornish and Hellings [9], in this situation it is necessary to keep track of the order of retardations: for instance, the unequalarm Michelson combination of Fig. 4 would translate to

\footnotetext{
${ }^{8}$ With second-generation TDI, the cancellation occurs up to (and including) terms proportional to $L_{l} \dot{L}_{m}$, which is more than sufficient for realistic LISA spacecraft orbits.
}

$$
\begin{array}{r}
y_{13^{\prime} 2 ; 322^{\prime}}(t)+y_{231 ; 22^{\prime}}(t)+y_{123 ; 2^{\prime}}(t)+y_{32^{\prime} 1}(t) \\
-y_{231}(t)-y_{13^{\prime} 2 ; 3}(t)-y_{32^{\prime} 1 ; 3^{\prime} 3}(t)-y_{123 ; 2^{\prime} 3^{\prime} 3}(t),
\end{array}
$$

where, using the semicolon notation of Ref. [10],

$$
\begin{aligned}
y_{i l j ; r_{1}}(t) & =y_{i l j}\left(t-L_{r_{1}}\right), \\
y_{i l j ; r_{2} r_{1}}(t) & =y_{i l j}\left(t-L_{r_{1}}-L_{r_{2}}\left(t-L_{r_{1}}\right)\right),
\end{aligned}
$$

and so on: the nominal time $t$ is delayed incrementally starting from the rightmost delay index $r_{1}$. (A similar notation with commas instead of semicolons is used when the armlengths are constant and the order of the retardations is not important.)

Inserting the laser noises $C_{i}$ in Eq. (9), we see that they cancel in pairs, except for the terms from the tails of the two paths,

$$
C_{1 ; 3^{\prime} 322^{\prime}}-C_{1 ; 22^{\prime} 3^{\prime} 3}(t) \simeq \dot{C}_{1}(t)\left[t_{; 3^{\prime} 322^{\prime}}-t_{; 22^{\prime} 3^{\prime} 3}\right]
$$

Taylor-expanding the retardations to first order and keeping only linear terms in the $\dot{L}_{l}(t)$, we get

$$
\dot{C}_{1}(t)\left[\left(L_{2^{\prime}}+L_{2}\right)\left(\dot{L}_{3}+\dot{L}_{3^{\prime}}\right)-\left(L_{3}+L_{3^{\prime}}\right)\left(\dot{L}_{2^{\prime}}+\dot{L}_{2}\right)\right]
$$

where all the $L_{l}$ and $\dot{L}_{l}$ are implicitly evaluated at time $t$. (More generally, each retardation index $r_{i}$ generates a residual term proportional to $L_{r_{i}} \dot{L}_{r_{j}}$ for each index $r_{j}$ to its left.)

In short, much like what happened with the simple Michelson combination [Eq. (7)] for unequal-arm geometries, a laser-noise residual appears in Eq. (12) because the light-travel times built up along the two interfering paths are different; graphically, the tails of the two 
paths do not match precisely. This is because, although both paths contain the same set of links, they do so in different orders, and the retardations do not commute when the armlengths are time dependent. (This is also the reason why the algebraic approach becomes arduous for second-generation TDI, where it involves the solution of polynomial equations for noncommuting variables.) As in the upgrade from equal-arm to unequal-arm (first-generation) Michelson observables, one solution is to compose the two paths so that each goes through each arm twice, in different orders [10]. The residual of the resulting 16-link combination vanishes up to the first Taylor order and to the first degree in $\dot{L}_{l}$ (henceforth, to first order/degree). This second-generation unequal-arm Michelson observables (known as $X_{1}$ ) may be written in our notation

$$
\begin{aligned}
& y_{13^{\prime} 2 ; 322^{\prime} 22^{\prime} 3^{\prime} 3}(t)+y_{231 ; 22^{\prime} 22^{\prime} 3^{\prime} 3}(t) \\
+ & y_{123 ; 2^{\prime} 22^{\prime} 3^{\prime} 3}(t)+y_{32^{\prime} 1 ; 22^{\prime} 3^{\prime} 3}(t) \\
+ & y_{123 ; 2^{\prime} 3^{\prime} 3}(t)+y_{32^{\prime} 1 ; 3^{\prime} 3}(t)+y_{13^{\prime} 2 ; 3}(t)+y_{231}(t) \\
- & y_{32^{\prime} 1}(t)-y_{123 ; 2^{\prime}}(t)-y_{231 ; 22^{\prime}}(t)-y_{13^{\prime} 2 ; 322^{\prime}}(t) \\
- & y_{231 ; 3^{\prime} 322^{\prime}}(t)-y_{13^{\prime} 2 ; 33^{\prime} 322^{\prime}}(t) \\
- & y_{32^{\prime} 1 ; 3^{\prime} 33^{\prime} 322^{\prime}}(t)-y_{123 ; 2^{\prime} 3^{\prime} 33^{\prime} 322^{\prime}}(t),
\end{aligned}
$$

which is related to the $X_{1}$ defined in Refs. 10, 11] by a change of sign and by the use of the opposite convention for primed and unprimed indices.

Second-generation generalizations of all firstgeneration TDI observables were described by Tinto and colleagues [10, 11]. For the analogs of the $\alpha, \beta$, $\gamma$, and $\zeta$ observables (which are formally identical to their modified TDI counterparts [8, 9], except for the interpretation of the delay indices as noncommuting), laser-noise cancellation is not complete, even to first order/degree: however, the residuals consist of symmetric sums of $L_{l} \dot{L}_{m}$ terms that turn out to be small for realistic LISA orbits.

\section{E. The combinatorial enumeration of TDI observables}

Our geometric approach to TDI makes it possible to enumerate all the second-generation TDI observables of given length. The key to this is the

Feynman-Wheeler ${ }^{9}$ Geometric TDI principle: any

\footnotetext{
9 "I received a telephone call one day at the graduate college at Princeton from Professor Wheeler, in which he said, 'Feynman, I know why all electrons have the same charge and the same mass.' 'Why?' 'Because, they are all the same electron!' [...] I did not take the idea that all the electrons were the same one from him as seriously as I took the observation that positrons could simply be represented as electrons going from the future to the past in a back section of their world lines." [R. P. Feynman, "The Development of the Space-Time View of Quantum Electrodynamics," Nobel Lecture, Dec 11, 1965.]
}

$2 N$-beam Geometric-TDI closed loop can be seen as a single beam that travels forward and backward in time to meet itself back at its origin.

For instance, the two-beam equal-arm-Michelson combination of Fig. 3 (left panel), can be interpreted as a single beam that departs at the initial time $t-L_{3}-L_{3^{\prime}}$, travels forward in time to be measured at time $t-L_{3}$, and again travels forward in time to be measured (and interfere against itself!) at time $t$; the beam then moves backward in time to be emitted at time $t-L_{2^{\prime}}$, and again moves backward in time to be emitted at the original time $t-L_{2^{\prime}}-L_{2}$ (equal to $t-L_{3}-L_{3^{\prime}}$, since the armlengths are equal). This closes the loop, and cancels laser noise at all junctions (when we translate graphs to formulas, we must remember to give minus signs to all the backward-time arrows, drawn dashed in Fig. 3 .

Once we have established that all $n$-link loops can be represented as a single loop, we can enumerate them combinatorially by choosing a starting spacecraft and, for $n$ times over, choosing the future or past time direction, and the leftward (clockwise) or rightward (counterclockwise) movement direction, in all possible combinations. Each loop can be denoted by the index of the initial spacecraft, followed by a string of "L" or "R" crested by " $\rightarrow$ " for forward-time arrows and by " $\leftarrow$ " for backward-time arrows; this notation is translated easily into strings of link indices crested by their time directions (henceforth, link strings). For instance, we would write (1) $\overrightarrow{\mathrm{LR}} \overleftarrow{\mathrm{LR}} \equiv \overrightarrow{3^{\prime} 32^{\prime} 2}$ and (1) $\overrightarrow{\mathrm{LRRLRLLR}} \equiv \overrightarrow{3^{\prime} 322^{\prime}} \overleftarrow{33^{\prime} 2^{\prime} 2}$ for the loops in the left and right panels of Fig. 3. respectively.

Not all $3 \times 2^{2 n}$ strings with $n$ links correspond to lasernoise-canceling combinations, because the total lighttravel time accumulated across the loop must be zero (for second-generation TDI, zero to first order/degree). However, it is quite straightforward to set simple closure criteria that identify the true TDI combinations:

Pre-TDI interferometry. For equal-arm geometries, the loop must end at the initial spacecraft $(\#[\overrightarrow{\mathrm{L}}, \overleftarrow{\mathrm{R}}]-$ $\#[\overrightarrow{\mathrm{R}}, \overleftarrow{\mathrm{L}}] \bmod 3=0$, where \# denotes the number of occurrences of a symbol in the string), and it must have a null total light-travel time $(\#[\overrightarrow{\mathrm{L}}, \overrightarrow{\mathrm{R}}]=\#[\overleftarrow{\mathrm{L}}, \overleftarrow{\mathrm{R}}])$. We denote the combinations that satisfy this property as closed.

First-generation TDI. For unequal-arm geometries with generic $L_{l}=L_{l^{\prime}}$, the loop must end at the initial spacecraft, and satisfy $\#\left[\vec{l}, \overrightarrow{l^{\prime}}\right]=\#\left[\overleftarrow{l}, \overleftarrow{l^{\prime}}\right]$ (for $l=1,2,3)$, which yields a null total light-travel time. We denote the combinations that satisfy this property as $|L|$-closed.

Modified TDI. For unequal-arm geometries with generic $L_{l} \neq L_{l^{\prime}}$, the loop must end at the initial spacecraft and satisfy $\#[\vec{l}]=\#[\overleftarrow{l}]$ (for $\left.l=1,1^{\prime}, 2,2^{\prime}, 3,3^{\prime}\right)$ which yields a null total light-travel time. We denote the 
combinations that satisfy this property as $L$-closed.

Second-generation TDI. For unequal-arm geometries with generic, time-dependent $L_{l}(t) \neq L_{l^{\prime}}(t)$, firstorder/degree laser-noise cancellation is obtained for loops that are $L$-closed, and in addition satisfy $\#[\vec{l} \vec{m}, \overleftarrow{l} \overleftarrow{m}]=$ $\#[\vec{l} \overleftarrow{m}, \overleftarrow{l} \overrightarrow{\dot{m}}]$ (with $l, m=1,1^{\prime}, 2,2^{\prime}, 3,3^{\prime}$ ), where a pair $l \dot{m}$ is counted for each link $\vec{l}$ with itself and with all the links $\vec{m}$ and $\overleftarrow{m}$ to its right, and for each link $\overleftarrow{l}$ with all the links $\vec{m}$ and $\overleftarrow{m}$ to its right. This condition yields a total light-travel time that is null to first order/degree (see App. A1). For instance, for $\overrightarrow{3^{\prime} 32^{\prime} 2}$ we count $\overrightarrow{3^{\prime} 3^{\prime}}, \overrightarrow{3^{\prime} 3}$, $\overrightarrow{3^{\prime}} \overleftarrow{2^{\prime}}, \overrightarrow{3^{\prime}} \overleftarrow{2}, \overrightarrow{33}, \overrightarrow{3} \overleftarrow{2^{\prime}}, \overrightarrow{3} \overleftarrow{2}$, and $\overleftarrow{2^{\prime} 2^{\prime}}$; hence the counting does not satisfy the property given above. We denote the combinations that satisfy this property as $\dot{L}$-closed.

The closure criteria induce useful symmetry properties for the link strings:

Null bigrams. The bigrams $\vec{L} \overleftarrow{L}, \vec{R} \overleftarrow{R}, \overleftarrow{L} \vec{L}$, and $\overleftarrow{R} \vec{R}$ (or equivalently $\vec{l} \overleftarrow{l}$ and $\overleftarrow{l} \vec{l}$ ) always represent combinations of two $y_{i j}$ measurements that sum up to exactly zero: any $n$-link string that contains such bigrams represents a combination of length smaller than $n$.

Cyclic string shift. Shifting a string cyclically produces a combination that has the same closure properties as the original, and that differs only by an overall time advancement or retardation, and by an advancement or retardation applied selectively to the shifted terms, but considered negligible at that closure level (for instance, for an $L$-closed loop the additional selective retardation would be of leading order/degree $L_{l} \dot{L}_{m}$; for an $\dot{L}$-closed loop, of a higher order/degree).

Time and direction reversal. Simultaneously swapping the primedness and time direction of all link indices produces a combination that has the same closure properties as the original, and that differs from the original by its handedness.

Cyclic index shift. Shifting link indices cyclically $(1 \rightarrow 2,2 \rightarrow 3,3 \rightarrow 1)$ produces a combination that has the same closure properties as the original, and that differs only by a relabeling of indices. Noncyclic index permutations, on the other hand, produce illegal strings (i.e., unconnected loops) unless all indices change in primedness, in time direction, or in a combination of the two. ${ }^{10}$

String reversal. Reversing a string while swapping all " $\rightarrow$ " and " $\longleftarrow$ " symbols (i.e., reversing all time directions) produces a combination that has the same closure properties as the original, and that differs only in sign and by

10 The reflection-like symmetry considered below Eq. [36] consists in exchanging two indices and swapping their primedness, while reversing the time direction of the third index. an overall time advancement or retardation considered negligible at that closure level.

Splicing. Inserting any link string at any compatible point within a link string yields another legal link string. Here compatibility means that the spacecraft visited by the first loop at the insertion point (for instance, after $\overrightarrow{1}$, spacecraft 2 ; after $\overleftarrow{1}$, spacecraft 3 ) must be the same as the initial spacecraft of the inserted loop. The resulting string has at least the closure properties shared by the spliced fragments.

For instance, the unequal-arm Michelson ( $L$-closed) string

$$
\overrightarrow{{ }_{1}{ }^{\prime}{ }_{2} 3_{1} 2_{3} 2^{\prime}}{ }_{1} \overleftrightarrow{3_{2} 3^{\prime}{ }_{1} 2^{\prime}{ }_{3} 2_{1}}
$$

(where the subscripts show the spacecraft visited by the loop before and after traversing each link) can be spliced at its center with its own $L$-closed reversal,

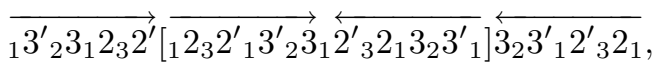

yielding an $\dot{L}$-closed loop that is in fact the secondgeneration TDI Michelson observable.

At this point it is also useful to give a rule to translate link strings into $y_{i j}$ combinations:

1. Starting at the left end of the string, write a $y_{i j}$ measurement for each index, according to the replacement rules $\left\{1,2,3,1^{\prime}, 2^{\prime}, 3^{\prime}\right\} \equiv$ $\left\{y_{32}, y_{13}, y_{21}, y_{23}, y_{31}, y_{12}\right\}$; attribute a plus sign for " $\rightarrow$ " links and a minus sign for " $\longleftarrow$ " links;

2. while doing this, build the delay sequence to be applied to each new $y_{i j}$, adding (from the left) an advancement index $\bar{r}$ before translating each $\vec{r}$, and a retardation index $s$ after having translated each $\overleftarrow{s}$

For instance, the $\stackrel{3^{\prime} 322^{\prime}}{33^{\prime} 2^{\prime} 2}$ string would translate to

$$
\begin{aligned}
& y_{13^{\prime} 2 ; \overline{3^{\prime}}}(t)+y_{231 ; \overline{33^{\prime}}}(t) \\
& +y_{123 ; \overline{233^{\prime}}}(t)+y_{32^{\prime} 1 ; \overline{2^{\prime} 233^{\prime}}}(t)
\end{aligned}
$$

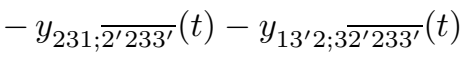

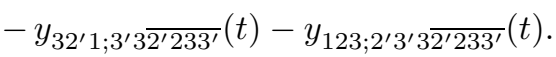

To accommodate advancement indices, the semicolon notation of Eq. (10) is extended by the advancement rule

$$
y_{i l j ; \bar{a}}(t)=y_{i l j}\left(t+\Gamma_{a}\right),
$$

with $\Gamma_{a}$ defined as the time experienced by light propagating along link $a$ for emission at time $t$, and given implicitly in terms of $L_{a}(t)$ by $\Gamma_{a}(t)=L_{a}\left(t+\Gamma_{a}(t)\right)$. Retardations and advancements are applied incrementally starting from the rightmost index.

Equation (16) appears more complicated than Eq. (9), but it encodes essentially the same $y_{i j}$ combination: the 
two equations are related by a time retardation, as can be seen by evaluating Eq. (16) at the time $t_{; 3^{\prime} 322^{\prime}}$. This adds $3^{\prime} 322^{\prime}$ to the right of the delay sequence for each $y_{i j}$, and since adjacent pairs $\bar{l} l$ and $l \bar{l}$ cancel in delay sequences (by the very definition of $L_{l}$ and $\Gamma_{l}$ ), Eq. (16) then turns into Eq. (9). Conversely, Eq. (13) may be obtained by applying the translation rule to the string $\overrightarrow{3^{\prime} 322^{\prime} 22^{\prime} 3^{\prime} 32^{\prime} 233^{\prime} 33^{\prime} 2^{\prime} 2}$, and evaluating the resulting expression at the time $t_{; 33^{\prime} 2^{\prime} 22^{\prime} 233^{\prime}}$. A slightly more complicated version (see App. A2 of the rule given above yields $y_{i j}$ combinations that are closer to standard TDI notation.

\section{F. Extension of Geometric TDI to six-laser LISA configurations}

The extension of our discussion to LISA configurations with six lasers introduces three additional laser phase noise variables $C_{1}^{*}(t), C_{2}^{*}(t)$, and $C_{3}^{*}(t)$, corresponding in Figs. 2] and 3 to the optical benches on the right-hand side of the spacecraft (if we look toward the center). Equation (6) changes accordingly:

$$
\begin{aligned}
& y_{i j}=C_{i ; l}^{*}-C_{j}+\mathrm{GWs} \quad \text { for unprimed } l(i, j), \\
& y_{i j}=C_{i ; l}-C_{j}^{*}+\mathrm{GWs} \quad \text { for primed } l(i, j) ;
\end{aligned}
$$

here unprimed and primed link indices $l(i, j)$ correspond to c.c.w. and c.w. $y_{i j}$ measurement directions, respectively. As shown in Ref. [11], all arguments and derivations valid with three lasers can be applied to a six-laser configuration by replacing all $y_{i j}$ with $y_{i j}^{(6)}\left(\eta_{i j}\right.$ in Ref. [11]) defined by

$$
\begin{array}{lr}
y_{i j}^{(6)}=y_{i j}-\frac{1}{2}\left(z_{l j ; l}-z_{i j ; l}\right) & \text { for unprimed } l(i, j), \\
y_{i j}^{(6)}=y_{i j}+\frac{1}{2}\left(z_{|l| j}-z_{i j}\right) & \text { for primed } l(i, j),
\end{array}
$$

where the $z_{i j}(t)$ are the back-plane measurements comparing the phases of the two lasers on each spacecraft, and where the notation $|l|$ removes a prime from $l$, if present. Because $z_{i j}(t)= \pm\left[C_{j}^{*}(t)-C_{j}(t)\right]$ [for unprimed and primed $l(i, j)$, respectively], the $C_{j}^{*}$ disappear from Eq. (18), and Eq. (6) is restored for the $y_{i j}^{(6)}$. This justifies all the developments reported in this paper also for six-laser LISA configurations.

It should be mentioned in this context that the phase noise from the random motion of the optical benches enters the $y_{i j}^{(6)}$ with the same time signature as the laser phase noises, and is therefore also canceled by TDI. The LISA sensitivity to GWs is then set by the remaining secondary noises. Adopting the schematization of the measurement process used in most of the TDI literature, and the notation used to describe the Synthetic LISA simulator [20], the response of the $y_{i j}^{(6)}$ to the secondary noises is given by

$$
\begin{array}{lr}
y_{i j}^{(6)}=y_{i j}^{\mathrm{op}}-2 p m_{j}-p m_{i ; l}^{*}+p m_{i ; l} & \text { for unprimed } l(i, j), \\
y_{i j}^{(6)}=y_{i j}^{\mathrm{op}}-p m_{j}^{*}-p m_{j} & \text { for } \operatorname{primed} l(i, j),
\end{array}
$$

where $y_{i j}^{\mathrm{op}}$ is the optical-path noise in the $y_{i j}$ phase measurement, and $p m_{i}$ and $p m_{i}^{*}$ are the velocity noises of the two proof masses aboard spacecraft $i$. Because $p m_{i ; l}-p m_{i}$ and $p m_{i ; l}^{*}-p m_{i}^{*}$ have the same time signature as laser phase noises, they are canceled in TDI observables; thus, all retardations can be removed from the unprimed- $l(i, j)$ expression of $y_{i j}^{(6)}$, casting it to the same form as its primed- $l(i, j)$ counterpart.

\section{A GEOMETRIC SURVEY OF SECOND-GENERATION TDI OBSERVABLES}

I have written a computer program to list all the second-generation TDI observables consisting of 24 or fewer $y_{i j}$ measurements. For each even length $n$, this was achieved by enumerating all $2^{2 n}$ possible LR strings, and checking each of them for $\dot{L}$-closure, according to the counting rule given in Sec. IIE Already for 24-link strings, the combinatorial space is huge, and an exhaustive search required more than 10,000 CPU hours. The resulting list of observables was then reduced to a minimal set by removing all the quasi-duplicates that differ only by a sign or by a cyclic string shift. I have kept as distinct the observables that differ by a cyclic index shift (in first-generation TDI, this would correspond to counting $X, Y$, and $Z$ as separate observables). The reduced list of TDI observables is available at the webpage www.vallis.org/tdi annotated with their temporal footprint (see Sec. IIIB), number of beams, type, and splicing composition (see Secs. [IIA and IIIC).

My results are tallied in Table प Here the $X, U, E$, and $P$ types represent generalizations of the 8 -link observables of the same name: $X$-type (Michelson) observables use two arms in both directions, $U$-type observables use four oriented arms in a relay configuration, E-type and $P$-type use four oriented arms in beacon and monitor configurations; the observables tallied under other use either five or six oriented arms. Here are the highlights of the survey, which are discussed in more detail in the following sections.

- I find that the shortest second-generation TDI observable has length 16. By contrast, modified-TDI observables begin at length 8 .

- I recover all ${ }^{11}$ the known 16-link second-generation TDI observables, previously obtained by Tinto and

\footnotetext{
${ }^{11}$ In fact, our $U$-type combinations do not include the $U_{1}$ observable given in Ref. 11], which achieves laser-noise cancellation at the approximate time $t-4 L$ through the sum of four distinct
} 


\begin{tabular}{r||c|r||r|r|r|r|r|r|r|r|r|r} 
links & comb. space & unique obs. & $X$-type & $P, E$-type & $U$-type & other & 2-beam & 4-beam & 6-beam & 8-beam & 10-beam and higher \\
\hline 16 & $4 \times 10^{9}$ & 48 & 12 & 18 & 18 & 0 & 3 & 27 & 0 & 18 & 6 \\
18 & $7 \times 10^{10}$ & 192 & 0 & 12 & 12 & 168 & 6 & 24 & 102 & 60 & 0 \\
20 & $1 \times 10^{12}$ & 660 & 24 & 18 & 18 & 600 & 12 & 114 & 276 & 90 & 0 \\
22 & $2 \times 10^{13}$ & 2412 & 0 & 36 & 36 & 2340 & 30 & 264 & 732 & 792 & 168 \\
24 & $3 \times 10^{14}$ & 12585 & 144 & 90 & 90 & 12261 & 99 & 945 & 2676 & 4566 & 594 \\
& & &
\end{tabular}

TABLE I: Geometric survey of second-generation TDI observables. Quasi-duplicates that differ only by a sign or by a time advancement are not counted. The $X, U, E$, and $P$ types represent generalizations of the 8-link observables of the same name: $X$-type (Michelson) observables use two arms in both directions, $U$-type observables use four oriented arms in a relay configuration, E-type and $P$-type use four oriented arms in beacon and monitor configurations; the observables reported under other use either five or six oriented arms. The number of beams corresponds to the number of contiguous substrings with the same time direction, minimized with respect to cyclic string shifts. All the observables tallied in this table are available in extenso at the webpage ww. vallis.org/tdi

colleagues [10, 11] by applying commutator-like delay operators to the 8-link observables of modified TDI. From a geometric viewpoint, all the 16link observables can be understood as self-splicings of the 8-link observables of the same type. This shows that the former reduce to finite time differences of the latter, up to time shifts of the first order/degree. It follows that the second-generation TDI observables have the same sensitivity of the modified TDI observables of the same type, ${ }^{12}$ not only in the equal-arm limit, but unconditionally.

See Sec. IIA for details.

- In addition, I obtain alternative forms of the known 16-link observables. The alternative forms use a larger number of beams (e.g., four beams for $X$, as opposed to the standard two), or a different allocation of links in the beams (e.g., $5+4+3+4$ or $6+4+2+4$ for $U$, as opposed to the standard $7+4+1+4$ structure). The alternative forms, too, can be understood as self-splicings of the 8link modified TDI observables of the same kind.

The alternative forms have the same sensitivity to GW signals as the original forms in idealized measurement conditions, but they can improve on them when realistic aspects (such as quantization of the phasemeter output and technical noises) are taken into account. In addition, the alternative forms have a reduced temporal footprint (the difference between the times of the earliest and latest phase measurements involved in their construction); this feature can be advantageous in the presence of gaps or glitches in the $y_{i j}$ data, because it reduces the extent of defect propagation to the TDI time series.

$y_{i j}$ measurements; by contrast, in Geometric TDI laser noise is always canceled by construction between pairs of phase measurements. However, the $U_{1}$ of Ref. [1] has almost the same temporal structure as our $\overline{1^{\prime} 12^{\prime} 3^{\prime}} \stackrel{\leftarrow}{2^{\prime} 3^{\prime} 1^{\prime} 13^{\prime} 2^{\prime} 1^{\prime} 11^{\prime} 3^{\prime} 2^{\prime}}$ (keep in mind that the primedness of our indices is the opposite of Ref. [11]).

12 Neglecting of course the fact that the modified-TDI observables would not cancel laser noise in a flexing LISA.
See Secs. IIIA and IIIB for details. Appendix A4 gives explicit algebraic expressions for all the 16link observables in terms of the $y_{i j}$ measurements.

- Second-generation TDI observables are found in increasing numbers at lengths $18,20,22$, and 24 . A minority are of the $X, U, E$, or $P$ types, while most use either five or six oriented arms.

All 18-to-24-long observables can be understood as splicings of modified-TDI observables of length 8 to 18 , sometimes with the inclusion of null bigrams; most, but not all, are self-splicings. I conjecture that all second-generation TDI observables of any length can be generated as splicings of two modified-TDI observables.

See Sec. IIIC for details.

- Up to length 24 , I do not find any $\dot{L}$-closed observables of the $\zeta$ type (defined as having suppressed, but nonzero, GW response at low frequencies). I conjecture that the $\zeta$ type is incompatible with $\dot{L}$-closure. This does not exclude the existence of non- $\dot{L}$-closed $\zeta$-type observables (such as the $\zeta_{1}, \zeta_{2}$, and $\zeta_{3}$ described by Tinto and colleagues [11]) that do not cancel laser noise to first order/degree, but bring it sufficiently below the LISA secondary noise to be useful in practice.

\section{A. Structure and sensitivity of the 16-link observables}

The standard second-generation TDI $X$ observable is

$$
X_{1}^{16,2}: \longdiv { 3 ^ { \prime } 3 2 2 ^ { \prime } 2 2 ^ { \prime } 3 ^ { \prime } 3 2 ^ { \prime } 2 3 3 ^ { \prime } 3 3 ^ { \prime } 2 ^ { \prime } 2 } \text { (2 beams) }
$$

which is related to the $X_{1}$ defined in Eq. (13) by $X_{1}^{16,2} \equiv$

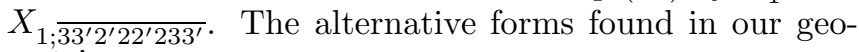
metric survey are

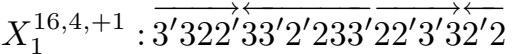

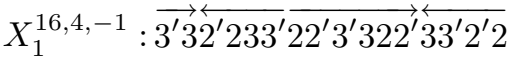

$$
\begin{aligned}
& \text { (4 beams), }
\end{aligned}
$$


which differ between themselves only by handedness, and

$$
X_{1}^{16,4,0}: \overrightarrow{22^{\prime} 22^{\prime}} \overleftrightarrow{33^{\prime} 2^{\prime} 2} \overrightarrow{3^{\prime} 33^{\prime} 32^{\prime} 233^{\prime}} \quad \text { (4 beams) }
$$

which turns out to have vanishing response in the equalarm limit to both noise and GWs, at all frequencies. To see that $X_{1}$ and the $X_{1}^{16,4, \pm 1}$ have all the same GW sensitivity as the 8-link modified-TDI $X$ (neglecting of course the fact that $X$ would not cancel laser noise in a flexing LISA), we reason as follows.

As we have learned in Eq. (15), $X_{1}$ can be interpreted as a self-splicing of $X$ with its reversal. If we take $X$ to be defined by Eq. (9), we see that

$$
\begin{aligned}
& \overrightarrow{3^{\prime} 322^{\prime}} \overleftrightarrow{33^{\prime} 2^{\prime} 2} \equiv X_{; \overline{2^{\prime}} 233^{\prime}} \\
& \stackrel{22^{\prime} 3^{\prime} 32^{\prime} 233^{\prime}}{\equiv}-X_{; \overline{33^{\prime} 2^{\prime} 2}}
\end{aligned}
$$

Since the time at the splicing point in $\overrightarrow{3^{\prime} 322^{\prime}} \overleftrightarrow{33^{\prime} 2^{\prime} 2}$ is $t_{; \overline{2^{\prime}} 233^{\prime}}$, from Eq. (15) we see that

$$
\overrightarrow{3^{\prime} 322^{\prime} 22^{\prime} 3^{\prime} 32^{\prime} 233^{\prime} 33^{\prime} 2^{\prime} 2} \simeq X_{; \overline{2^{\prime}} 233^{\prime}}-X_{; 33^{\prime} 2^{\prime} 22^{\prime} 233^{\prime}}
$$

here the symbol " $\simeq "$ denotes equality up to selective delays or advancements of order $L_{l} \dot{L}_{m}$ in the $y_{i j}$, not specified by the formal delay strings. [In this case, these spurious delays appear because $\overrightarrow{22^{\prime} 3^{\prime} 3} \overleftrightarrow{2^{\prime} 233^{\prime}}$ is only $L$ closed, so the time at the beginning and at the end of the inserted string is different by terms of order $L_{l} \dot{L}_{m}$; consequently, the last four $y_{i j}$ observables of $\overrightarrow{3^{\prime} 322^{\prime} 33^{\prime} 2^{\prime} 2}$ are really evaluated at the time $t_{; 3^{\prime} 322^{\prime}} \overline{33^{\prime} 2^{\prime} 22^{\prime} 233^{\prime}}$, not just $t_{\left.; \overline{2^{\prime} 233^{\prime}} .\right]}$

Rewriting Eq. (25) in terms of $X_{1}$ and reabsorbing the time advancements by evaluating the equation at time $t_{; 3^{\prime} 322^{\prime} 22^{\prime} 3^{\prime} 3}$, we find

$$
X_{1} \simeq X_{; 22^{\prime} 3^{\prime} 3}-X
$$

Thus, up to delays of first order/degree, self-splicings produce finite differences of observables. (Indeed, it is a well-known fact in the literature on second-generation TDI observables that the standard 16-link observables are approximately equal to finite differences of the standard 8-link observables of the same type.)

Now, because the individual $y_{i j}$ measurements respond linearly ${ }^{13}$ to GWs and to all instrumental noise sources, the strain sensitivity of $X$ to monochromatic sources of frequency $f$ at a given sky position is proportional to

\footnotetext{
13 Linearity is always assumed in the model of measurement used to derive TDI. Significant nonlinearity in the phase measurements has been explored little, but would probably prove very detrimental to the delicate cancellation of laser phase noise achieved by TDI.
}

$\tilde{X}^{n}(f) / \tilde{X}^{\mathrm{GWs}}(f)$, where $\tilde{X}^{n}(f)$ is the (square-root) spectral density of noise in $X$, and $\tilde{X}^{\mathrm{GWs}}$ is the Fourier transform of the GW response function. The constant of proportionality is $\mathrm{SNR} / \sqrt{T_{\mathrm{obs}}}$, where $\mathrm{SNR}$ is the fiducial signal-to-noise ratio at which the sensitivity is defined, and $\sqrt{T_{\text {obs }}}$ is the time duration of the observation. Combining the Fourier-transform time-shifting property with Eq. (26), and considering that first order/degree terms can be neglected for secondary noises and GWs (which are much weaker than the laser phase noises), we see that $X_{1}$ must have the same sensitivity as $X$ :

$$
\frac{\tilde{X}_{1}^{n}(f)}{\tilde{X}_{1}^{\mathrm{GWs}}(f)}=\frac{\left(e^{2 \pi i f \Delta t}-1\right) \tilde{X}^{n}(f)}{\left(e^{2 \pi i f \Delta t}-1\right) \tilde{X}^{\mathrm{GWs}}(f)} \simeq \frac{\tilde{X}^{n}(f)}{\tilde{X}^{\mathrm{GWs}}(f)}
$$

with $\Delta t=t_{; 22^{\prime} 3^{\prime} 3}-t$. This is true generically for any spacecraft geometry, and not just in the equal-arm limit.

To see that $X_{1}^{16,4,+1}$ and $X_{1}^{16,4,-1}$, too, have the same sensitivity to GWs as $X$ (in the limit of perfect laser phase noise cancellation), it is then sufficient to show that they are self-splicings of $X$ and of its (cyclically shifted) reversal:

$$
\begin{aligned}
& X_{1}^{16,4,+1}: \overrightarrow{3^{\prime} 322^{\prime}\left[33^{\prime} 2^{\prime} 233^{\prime}\right.} \mid \overrightarrow{\left.22^{\prime}\right]} \overleftrightarrow{3^{\prime} 32^{\prime} 2} \text {, } \\
& X_{1}^{16,4,-1}: \overrightarrow{3^{\prime} 3}\left[\overleftrightarrow{2^{\prime} 233^{\prime}} \mid \overrightarrow{\left.22^{\prime} 3^{\prime} 3\right] 22^{\prime} 33^{\prime} 2^{\prime} 2}\right.
\end{aligned}
$$

Moving on to the $U$ type, we see (for instance) that the three second-generation $U$-type observables that use the oriented arms $1,1^{\prime}, 2$, and 3 ,

$$
\begin{aligned}
& \overrightarrow{3211^{\prime}} \stackrel{\overleftrightarrow{231}[23}{\left.1^{\prime} 1321^{\prime} 1\right] 1^{\prime}}, \\
& \overrightarrow{3211^{\prime} 23} \overleftarrow{\leftrightarrow}\left[1^{\prime} 1321^{\prime} 123\right] 11^{\prime}, \\
& 3211^{\prime}\left[132 \mid \overleftrightarrow{1^{\prime} 123} \overrightarrow{1^{\prime}}\right] \overleftrightarrow{2311^{\prime}},
\end{aligned}
$$

are generated by the self-splicings of the modified TDI $U$-type observable that uses the same oriented arms, $\overrightarrow{3211^{\prime}} 2311^{\prime}$. Thus, the modified TDI and secondgeneration TDI $U$-type observables have the same GW sensitivity (in the limit of perfect laser phase noise cancellation). Similar arguments hold for the $P$ - and $E$-type observables.

Altogether, we find empirically that all the 16-link second-generation TDI observables can be generated as self-splicings of the 8-link modified TDI observables of the same kind. Conversely, it can be proved that all selfsplicings of $L$-closed observables are $\dot{L}$-closed (see App. A3).

\section{B. Advantages of the alternative 16-link observables}

As mentioned above, the alternative forms of the 16link observables can have a smaller time footprint than the standard forms. For instance, the standard $X_{1}(t)$ of 


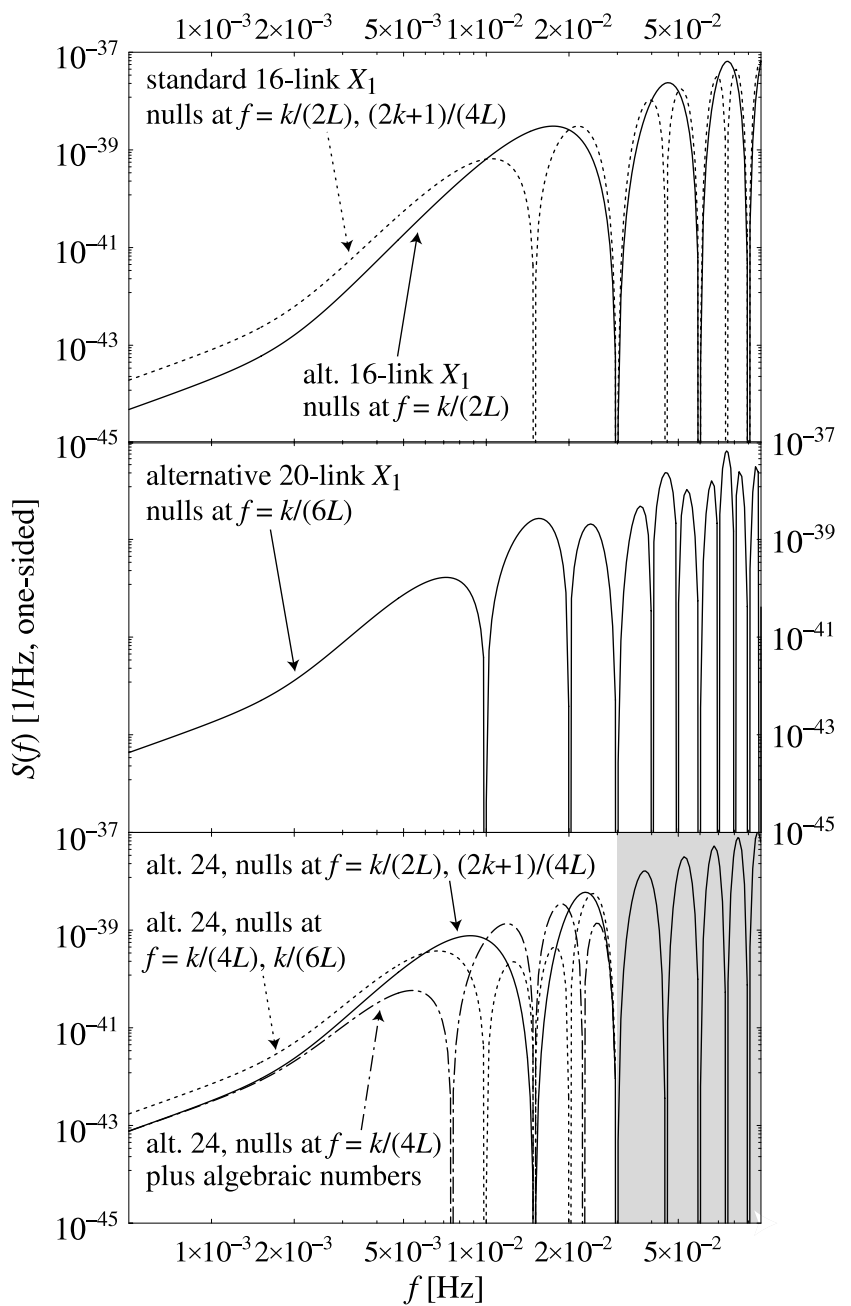

FIG. 5: TDI response of the second-generation $X$-type variables to the fundamental secondary noises, according to Eq. (20), assuming equal arms and proof-mass optical-path noise spectral densities given by $S_{i}^{\mathrm{pm}}=2.5 \times 10^{-48}[\mathrm{f} / \mathrm{Hz}]^{-2} \mathrm{~Hz}^{-1}$ and $S_{i}^{\text {op }}=1.8 \times 10^{-37}[\mathrm{f} / \mathrm{Hz}]^{2} \mathrm{~Hz}^{-1}$ (following Ref. 20]). The top panel shows the noise response of the standard 16-link $X_{1}$ observable, as compared to the response of the alternative 16link forms of Eq. 22, which have half as many nulls. The middle panel shows the noise response of the two-beam, 20link $X_{1}$ observables of Eq. (30). The bottom panel shows the three new noise responses found for 24-link $X_{1}$ observables (solid and dashed: self- and nonself-splicings of 12-link observables; dash-dotted: self-splicings of 8-link observables, with inclusions). To avoid visual clutter, two of the 24-link $X_{1}$ curves are not plotted beyond $f=1 /(2 L)$ (shaded region).

Eq. (13) involves $16 y_{i j}$ measurements taken ${ }^{14}$ within the interval $\left[\min \left(t_{; 3^{\prime} 322^{\prime} 22^{\prime} 3^{\prime} 3}, t_{; 22^{\prime} 3^{\prime} 33^{\prime} 322^{\prime}}\right), t\right]$, for a time span

14 Although the nominal times of the $y_{i j}$ are displaced only by $\simeq 7 L$, we must remember that in a six-laser LISA configuration some of the $y_{i j}^{(6)}$ contain one additional delay, as given by Eq. (19). $\simeq 8 L$, and each single $y_{i j}$ measurement appears in $X_{1}$ at times displaced by as much as $\simeq 6 L$. Thus, $X_{1}$ will be unavailable during the first and the last $\simeq 136$ s (i.e., $8 L$ ) within each LISA data-taking period. Moreover, a data gap in a single $y_{i j}$ measurements will appear in the $X_{1}$ time series at four distinct times spanning $\simeq 6 L$. By contrast, the alternative forms $X_{1}^{16,4, \pm 1}$ involve $16 y_{i j}$ measurements taken within a time interval of span $\simeq 6 L$, and the single $y_{i j}$ appear at times displaced by at most $\simeq 4 L$. The gain is significant, if not dramatic.

The alternative forms for the $U-, E$ - and $P$-type variables also yield footprints gains with respect to their standard forms (from $\simeq 7 L$ to $\simeq 5 L$ for $U$, and from $\simeq 5 L$ to $\simeq 4 L$ for $E$ and $P$ ). These gains are possible because the alternative observables are obtained, loosely speaking, by folding the standard versions in time, using both time advancements and retardations, as opposed to retardations only, to arrange the $y_{i j}$ measurements so that laser phase noise is canceled at all emission and reception events.

Another advantage of the alternative forms is that they can yield an improvement in GW sensitivity in realistic measurement conditions. In the top panel of Fig. [5] the dashed curve shows the power spectral density (PSD) of secondary noise for the standard $X_{1}$ observable, drawn in the limit of equal armlengths. Following Ref. 20], we assume that secondary noise consists entirely of proof-mass noise (idealized as stationary and Gaussian, with PSD $S_{i}^{\mathrm{pm}}=2.5 \times 10^{-48}[\mathrm{f} / \mathrm{Hz}]^{-2} \mathrm{~Hz}^{-1}$ ) and of optical-path noise (also stationary and Gaussian, with PSD $S_{i}^{\text {op }}=1.8 \times 10^{-37}[\mathrm{f} / \mathrm{Hz}]^{2} \mathrm{~Hz}^{-1}$ ). As discussed around Eq. 27), the sensitivity to GWs (averaged over noise realizations) is computed by dividing the secondary-noise rms power by the GW transfer function. ${ }^{15}$ See, for instance, Ref. [6] for plots of the GW sensitivities of the first-generation $X-, U_{-}, P-$, and $E$ type observables, common also (as discussed above) to the second-generation observables of the same type.

Although the noise PSD has nulls at multiples of the inverse armlength light-travel time [for the standard $X_{1}$, the nulls are at $f=k /(4 L)]$, the sensitivity to GWs remains finite in idealized conditions, because the GW transfer function displays zeros of the same order at the same frequencies. In reality, we should expect a degradation of sensitivity at these frequencies, because noise as a whole can only drop to the level of uncanceled laser noise, ${ }^{16}$ or of other technical noises (such as quantiza-

15 Roughly speaking, the GW transfer function for the standard $X_{1}$ is found by inserting Eq. (5) into Eq. 131 and Fouriertransforming, assuming a monochromatic source at a fixed sky location. The resulting transfer function is usually integrated over sky locations.

${ }^{16}$ However, the simulations of Ref. 23] seem to suggest that uncanceled laser noise, under the assumption of perfectly linear responses, will display the same nulls as the fundamental secondary noises. 
tion noise), effectively filling in the nulls. The nulls are unwelcome also because they imply that a very large dynamic range is needed for sensitive measurement at those frequencies. The alternative forms $X_{1}^{16,4, \pm 1}$ improve on this situation, because their noise PSD and GW transfer functions ${ }^{17}$ have half as many nulls [at $f=k /(2 L)$ ], as shown by the solid curve in the top panel of Fig. 5]

Similar gains are found for the other 16-link secondgeneration TDI observables. The standard $U$-type observable of Ref. [11], which we may represent as ${ }^{18}$ $\overrightarrow{3211^{\prime} 1321^{\prime} 123} \overrightarrow{1^{\prime}} \overleftrightarrow{2311^{\prime}}$, has nulls at $f=k /(3 L)$, while the alternative form $\overrightarrow{3211^{\prime}} 231231^{\prime} 1321^{\prime} 11^{\prime}$ has nulls only at $f=k / L$. The distribution of the nulls improves also for all the alternative forms of the $E$-and $P$-type observables.

\section{Longer observables}

Although the size of the combinatorial space of LR strings scales as $2^{2 n}$ with increasing string length $n$, we find empirically that the number of valid $\dot{L}$-closed combinations grows only as $2^{n}$ (at least up to $n=24$ ); by contrast, the number of the less constrained $L$-closed combinations grows roughly as $2^{3 n / 2}$ (at least up to $n=16$ ).

The majority of the longer second-generation observables use either five or six LISA oriented arms, and therefore do not belong to any of the $X, U, E$, or $P$ types. We do however find new forms for these. Throughout this section, we shall consider examples of the $X$ type: findings and conclusions are similar for the other types.

At length 20, we find two $X$-type observables with two beams,

$$
\begin{aligned}
& \overrightarrow{3^{\prime} 322^{\prime} \mathbf{2 2 ^ { \prime }}\left[22^{\prime} 3^{\prime} 32^{\prime} 233^{\prime}\right] \mathbf{2}^{\prime} \mathbf{2} 33^{\prime} 2^{\prime} 2}, \\
& \overline{3^{\prime} 322^{\prime} \mathbf{3}^{\prime} \mathbf{3}\left[22^{\prime} 3^{\prime} 32^{\prime} 233^{\prime}\right] \mathbf{3 3}^{\prime} 33^{\prime} 2^{\prime} 2,}
\end{aligned}
$$

which can be interpreted as the self-splicing of the 8-link modified TDI observable $\overrightarrow{3^{\prime} 322^{\prime}} \overleftrightarrow{33^{\prime} 2^{\prime} 2}$ with its reversal $22^{\prime} 3^{\prime} 32^{\prime} 233^{\prime}$, after the insertion of the double null bigrams $\overrightarrow{22^{\prime}} \overleftarrow{2^{\prime} 2}$ and $\overrightarrow{3^{\prime} 333^{\prime}}$ [shown in bold in Eq. (30)]. Because the $y_{i j}$ measurements corresponding to the null bigrams sum up to zero to first order/degree, we can repeat the arguments of Sec. IIIA to see that the 20-link $X_{1}$ observables of Eq. (30) have the same sensitivity as the standard 16-link form. Moreover, reasoning along the lines of App. A3, we can prove that the self-splicings of $L$-closed strings are $\dot{L}$-closed even with the inclusion of null bigrams.

17 In the equal-arm limit, $S_{n}^{\text {orig, }, 16}=4 \cos ^{2}(2 \pi f L) S_{n}^{\text {alt, } 16}$.

18 As discussed in note 11 the observables are not identical: however, they do have the same secondary noise PSD and GW transfer function.
At length 20 we find also alternative $X_{1}$ observables with four beams,

$$
\begin{aligned}
& \overrightarrow{3^{\prime} 322^{\prime} \mathbf{2 2}}\left|\overleftrightarrow{33^{\prime}}\right| \overrightarrow{22^{\prime} 3^{\prime} 3} \overleftrightarrow{\left.2^{\prime} 2\right] \mathbf{2}^{\prime} \mathbf{2} 33^{\prime} 2^{\prime} 2} \\
& \overrightarrow{3^{\prime} 322^{\prime} \mathbf{3}^{\prime} \mathbf{3}\left[3^{\prime} 32^{\prime} 233^{\prime}\right.} \mid \overrightarrow{\left.22^{\prime}\right]} \overleftrightarrow{\mathbf{3 3}^{\prime} 33^{\prime} 2^{\prime} 2} \\
& \overrightarrow{3^{\prime} 33^{\prime} 3\left[22^{\prime} 3^{\prime} 32^{\prime} 233^{\prime}\right] \mathbf{3 3}^{\prime} 22^{\prime}} \overleftrightarrow{33^{\prime} 2^{\prime} 2}
\end{aligned}
$$

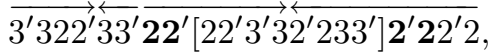

and with six beams,

$$
\begin{aligned}
& \overrightarrow{3^{\prime} 322^{\prime} 33^{\prime}} \overrightarrow{\mathbf{2 2}}\left[\overleftrightarrow{3^{\prime} 3} \mid \overrightarrow{\left.22^{\prime} 3^{\prime} 32^{\prime} 2\right] \mathbf{2}^{\prime} \mathbf{2} 2^{\prime} 2},\right.
\end{aligned}
$$

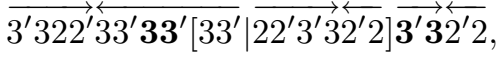

all of which are again self-splicings of $\overrightarrow{3^{\prime} 322^{\prime} 33^{\prime} 2^{\prime} 2}$ with two null-bigram inclusions. The secondary-noise PSD of the two-beam, 20-link $X_{1}$ observables of Eq. (30) is shown in the middle panel of Fig. [5] and has nulls at $f=k /(6 L)$. This PSD is not found for any of the 16-link $X_{1}$ observables. The four-beam observables have the secondarynoise PSD of the standard 16-link $X_{1}$ (the dashed curve in the top panel of Fig. 5), while the six-beam observables have the PSD of the alternative 16-link $X_{1}$ (the solid curve in the top panel of Fig. [5).

We find more $X$-type observables at length 24 . Some of these, such as

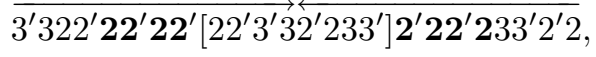

are self-splicings of the 8-link modified TDI $X$ with quadruple null-bigram inclusions, and have the same GW sensitivity as the standard 16-link $X_{1}$. They can have the same secondary-noise PSDs as the 16- and 20-link $X_{1}$ observables, or a new PSD (the dash-dotted curve in the bottom panel of Fig. 5ith nulls at $f=k /(4 L)$ and at frequencies given by third-degree algebraic numbers.

Other 24-link $X$-type observables, such as

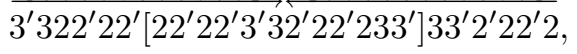

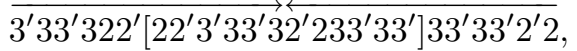

are self-splicings of the 12-link modified TDI $X$-type observables $\overrightarrow{3^{\prime} 322^{\prime} 22^{\prime}} \stackrel{\leftrightarrow 33^{\prime} 2^{\prime} 22^{\prime} 2}{\text { and }} \overrightarrow{3^{\prime} 33^{\prime} 322^{\prime}} \stackrel{\leftrightarrow 33^{\prime} 33^{\prime} 2^{\prime} 2}{ }$. The 24-link observables of Eq. (34) can be shown to have the same GW sensitivity as the standard 16-link $X_{1}$ in the equal-armlength limit. To see this, we parse the 8 - and 12 -link $X$-type variables as self-splicings of the pre-TDI (simply closed) observable $\overrightarrow{3^{\prime} 32^{\prime} 2}$, respectively without and with double-null-bigram inclusions:

$$
\begin{aligned}
& \overrightarrow{3^{\prime} 3\left[22^{\prime}\right.} \overleftrightarrow{\left.33^{\prime}\right] 2^{\prime} 2}, \\
& \overrightarrow{3^{\prime} 3 \mathbf{2 2} 2^{\prime}\left[22^{\prime}\right.} \stackrel{\left.33^{\prime}\right] \mathbf{2}^{\prime} \mathbf{2 2 ^ { \prime }} 2}{ } \\
& \overrightarrow{3^{\prime} 3 \mathbf{3}^{\prime} \mathbf{3}\left[22^{\prime}\right.} \overleftrightarrow{\left.33^{\prime}\right] \mathbf{3 3}^{\prime} 2^{\prime} 2} \text {. }
\end{aligned}
$$

If the armlengths are equal, the $y_{i j}$ measurements from the double bigrams shown in bold sum up to zero, and 
give no contribution to the GW and secondary-noise responses; if the armlengths are different, the presence of the bigrams introduces a preferred direction that distinguishes the sensitivities of the 8-link and 12-link observables. In the equal armlength limit, the observables of Eq. (34) can have the same secondary-noise PSD as the standard 16-link $X_{1}$ observables, or they can have two new PSDs (shown as solid and dotted curves in the bottom panel of Fig. (5) with nulls at $f=k /(2 L)$ and $f=(2 k+1) /(4 L)$, and at $f=k /(4 L)$ and $f=k /(6 L)$, respectively.

At length 24 we find also some $X$-type observables derived from non-self-splicings of 12 -link modified TDI $X$ type observables, such as

$$
\begin{aligned}
& \stackrel{3^{\prime} 322^{\prime} 22^{\prime}\left[22^{\prime} 3^{\prime} 33^{\prime} 32^{\prime} 233^{\prime} 33^{\prime}\right] 33^{\prime} 2^{\prime} 22^{\prime} 2,}{3^{\prime} 33^{\prime} 322^{\prime}\left[22^{\prime} 22^{\prime} 3^{\prime} 32^{\prime} 22^{\prime} 233^{\prime}\right] 33^{\prime} 33^{\prime} 2^{\prime} 2} .
\end{aligned}
$$

These non-self-splicings occur between 12-link $L$-closed link strings that differ by a reflection-like, noncyclic index shift (e.g., $2 \leftrightarrow 3^{\prime}, 3 \leftrightarrow 2^{\prime}$, with 1 and $1^{\prime}$ reversing time direction, if they are present in the string). In the equalarmlength limit, these observables have again the same sensitivity as the standard 16-link $X_{1}$, and they have the same secondary-noise PSDs as either the standard 16-link $X_{1}$ observables or the self-splicings of 12-link observables discussed above.

We now direct our consideration back to the full set of $\dot{L}$-closed link strings of lengths 16 to 24 . By exhaustive exploration, we were able to show that all of them can be obtained as splicings of two shorter $L$-closed link strings. An explicit specification of the splicings is given in the lists of TDI observables available at the webpage Www.vallis.org/tdi A good portion of the $\dot{L}$-closed link strings are obtained from self-splicings. The nonself-splicings occur between $L$-closed strings that are often related by evident symmetries (such as index shifts). Interestingly, these symmetries may occur between $L$ closed strings of different length, as it happens for the 20-link observable

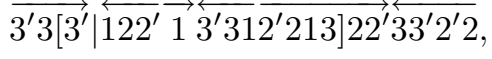

which can be interpreted as the splicing of the 8-link $X$ string $\overrightarrow{3^{\prime} 322^{\prime}} \stackrel{3}{33^{\prime} 2^{\prime} 2}$ with the reversal of the 12-link string

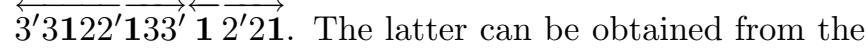
former by reversing the time direction of all $3^{\prime} 3$ and $22^{\prime}$ bigrams, and inserting $\overrightarrow{\mathbf{1}}$ and $\overleftarrow{\mathbf{1}}$ bridges as needed to keep the arrows consecutive, as shown in Fig. 6.

Also interesting is that some splicings result in $\dot{L}$-closed strings shorter than the sum of the lengths of their components, as in the case of the 22-link observable

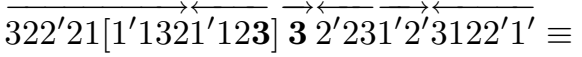

$$
\begin{aligned}
& \overrightarrow{322^{\prime} 211^{\prime} 1321^{\prime} 122^{\prime} 231^{\prime} 2^{\prime}} \overleftrightarrow{3122^{\prime} 1^{\prime}},
\end{aligned}
$$

which can be interpreted as the splicing of the 16link string $\overrightarrow{322^{\prime} 2132^{\prime} 231^{\prime} 2^{\prime}} \overleftrightarrow{3122^{\prime} 1^{\prime}}$ with the 8-link $U$-type
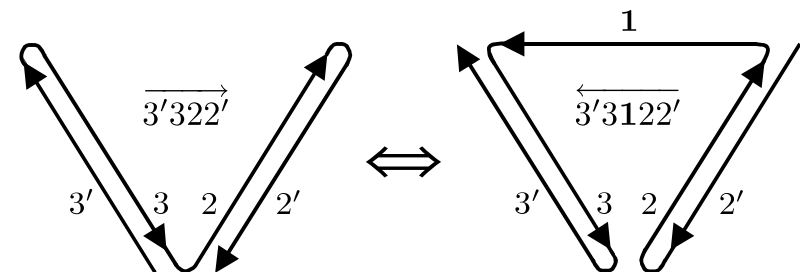

FIG. 6: The time-reversal plus bridge symmetry is exploited by certain $\dot{L}$-closed non-self-splicings at length 20 and above, such as the link string of Eq. (37).

string $\overrightarrow{1^{\prime} 132} \overleftrightarrow{1^{\prime} 123}$, occurring in such a way that the final $\overleftarrow{3}$ of the latter is erased by the subsequent $\overrightarrow{3}$ from the former [both bold in Eq. (38)].

We conjecture that all $\dot{L}$-closed strings can be obtained as splicings of $L$-closed strings. A possible proof could focus on the monadic $L$-closed strings (those that cannot be obtained as the splicing of two $L$-closed strings), which appear at every even length: consider for instance the string $\longdiv { 3 ^ { \prime } 3 2 2 ^ { \prime } 2 2 ^ { \prime } \cdots 2 2 ^ { \prime } } \overleftrightarrow { 3 3 ^ { \prime } 2 ^ { \prime } 2 2 ^ { \prime } 2 \cdots 2 ^ { \prime } 2 }$. To prove our conjecture, one would show that monadic $L$-closed strings are never $\dot{L}$-closed, as we know to be the case up to length 24.

\section{CONCLUSIONS}

I have described Geometric TDI, a powerful new approach to understand TDI intuitively, and to interpret all of its observables as the measurements of virtual synthesized interferometers, extending the original intuition of Tinto and Armstrong [4] and the graphical explanations of Shaddock 10] and Summers 14]. In Geometric TDI, observables consisting of $n$ one-way phase measurements are represented by link strings of length $n$, and can be enumerated exhaustively by listing all possible link strings, and then applying simple rules to determine which strings correspond to first-generation $(|L|$ closed), modified ( $L$-closed), or second-generation $(\dot{L}$ closed) observables. A study of the closure symmetries of link strings provides clues to the general rule that modified TDI observables combine (i.e., splice) into secondgeneration observables, maintaining in most cases the same GW sensitivity.

In addition to its pedagogical value, Geometric TDI has the practical interest of providing a systematic method to explore the space of second-generation TDI observables; such a method was unavailable prior to this work. Possible applications include the optimization of GW sensitivity (in analogy to the work of Prince and colleagues in Ref. [13]), and the development of targeted noise diagnostics.

In Sec. III I have used Geometry TDI to survey all second-generation TDI observables of lengths up to 24. My results (available in full at the webpage www.vallis.org/tdi) show that the garden of TDI observables contains a wealth of previously unknown spec- 
imens; this richness only increases with string length. In Sec. IIIB I have pointed out how certain alternative forms of the standard observables [such as the four-beam $X_{1}$ observables of Eq. [22)] have improved GW sensitivity in realistic measurement conditions (because they have fewer noise and GW response nulls), and reduced susceptibility to gaps and glitches (because they have a smaller temporal footprint).

The new observables become possible in Geometric TDI because the time advancements and retardations of one-way phase measurements are put on the same footing, while only retardations were considered in traditional TDI. This generalization does not constitute a third-generation TDI, but its combinatorial power and attractive symmetry justify its addition to the canon of TDI. There seem to be no particular challenges to implementing time-symmetric observables, especially in the framework of post-processed TDI [24], whether performed onboard or on the ground.

Future work on Geometric TDI should endeavor to prove the two open conjectures formulated in this paper: namely, that all second-generation TDI observables of any length can be generated as splicings of two modifiedTDI observables, and that there are no second-generation TDI observables of the perfect $\zeta$ type. (It would also be interesting to explore a notion of relaxed $\dot{L}$-closure that includes the $\zeta_{1}, \zeta_{2}$, and $\zeta_{3}$ variables described by Tinto and colleagues [11.).) Other promising directions of research include the optimization of GW sensitivity (can it be characterized geometrically?) and the exploration of generative rules other than splicing for second-generation TDI observables (what is the geometric counterpart and generalization of the algebra of observables studied in Refs. [7]?).

\section{Acknowledgments}

I wish to thank John Armstrong, Frank Estabrook, and Massimo Tinto for teaching me all things TDI; I thank also Daniel Shaddock and David Summers for useful discussions. I am grateful to the Supercomputing and Visualization Facility at the Jet Propulsion Laboratory for providing CPU time on their Orion cluster. This research was supported by the LISA Mission Science Office at the Jet Propulsion Laboratory, Caltech, where it was performed under contract with the National Aeronautics and Space Administration.

\section{APPENDIX A: RULES AND PROOFS}

\section{Counting rule for $\dot{L}$-closed link strings}

There is nothing magic about this rule, which is necessary and sufficient to have a null total light-travel time at first order/degree for generic armlengths $L_{l}(t)$. To see why, set $t=0$ at the beginning of the link string; stepping through the string, increment $t$ by an advancement $\Gamma_{l}(t)$ (defined as the time experienced by light propagating along link $l$ for emission at time $t$ ) for each symbol $\vec{l}$, and decrement $t$ by a delay $L_{l}(t)$ for each symbol $\overleftarrow{l}$. Each advancement $\Gamma_{l}$ or delay $L_{l}$ enters the time arguments of all subsequent $\Gamma_{m}$ 's and $L_{m}$ 's in the string, generating terms $\left\{\Gamma_{l},-L_{l}\right\} \times\left\{\dot{\Gamma}_{m},-\dot{L}_{m}\right\}$ in the firstorder/degree Taylor expansion of the total light-travel time. Since $\Gamma_{l}(t)=L_{l}\left(t+\Gamma_{l}(t)\right) \simeq L_{l}(t)+L_{l}(t) \dot{L}_{l}(t)$, we can replace all $\Gamma_{l}$ 's with $L_{l}$ 's (while keeping the overall sum the same to first order/degree) by adding a term $L_{l} \dot{L}_{l}$ for each $\vec{l}$ in the string. The counting rule given in the main text is then equivalent to requiring that all the $L_{l} \dot{L}_{m}$ terms cancel one by one to yield a null total light-travel time.

\section{Translation rule from link strings to quasi-standard TDI expressions}

1. Start at a bigram of type $\vec{l} \overleftarrow{m}$ (there must be at least one in every closed loop), shifting the string cyclically to move the bigram close to the middle;

2. move to the left, starting with $\vec{l}$, and write down a $y_{i j}$ measurement for each index, according to the replacement rules $\left\{1,2,3,1^{\prime}, 2^{\prime}, 3^{\prime}\right\} \equiv$ $\left\{y_{32}, y_{13}, y_{21}, y_{23}, y_{31}, y_{12}\right\}$; use a plus sign for “ $\rightarrow$ " links and a minus sign for " $\longleftarrow$ " links;

3. while doing this, build the delay sequence to be applied to each new $y_{i j}$, adding a retardation ; $r$ after having translated each $\vec{r}$, and an advancement $; \bar{s}$ before translating each $\overleftarrow{s}$;

4. after reaching the left end of the string, go back to the link index $\overleftarrow{m}$ in the initial bigram, and move to the right, using the same replacement rules;

5. while doing this, build the delay sequence from scratch, adding a retardation;s after having translated each $\overleftarrow{s}$, and an advancement ; $\bar{r}$ before translating each $\vec{r}$.

Shifting the string cyclically by a few positions can reduce the length of the delay sequence.

\section{Proof that all self-splicings of $L$-closed observables are $\dot{L}$-closed}

A few lemmas are needed for this proof. Looking back to the counting of $l \dot{m}$ pairs outlined in Sec. [IE we denote as prod[string] the polynomial obtained by regarding the 36 possible $l \dot{m}$ pairs as monomials, and summing them with coefficients \#[ $\overrightarrow{l \dot{m}}, \overleftarrow{l \dot{m}}]-\#[\vec{l} \overleftarrow{\dot{m}}, \overleftarrow{l} \overrightarrow{\dot{m}}]$. A string is $\dot{L}$-closed iff prod[string] $=0$. 
Lemma 1.-The prod of an $L$-closed string is unchanged after a cyclic string shift. Consider the shift of a single index $m$ from one end of the string to the other. Because the string is $L$-closed, and must therefore have $\#[\vec{l}]=\#[\overleftarrow{l}]$, the contribution to prod[string] from the index $m$ sums down to zero if $m$ carries a $\rightarrow$, or to $m \dot{m}$ if it carries a $\leftarrow$ (and therefore does not multiply itself). After the shift, the contribution to prod[shifted string] from the index $m$ is the same. Hence this lemma.

Lemma 2.-The prods of an $L$-closed string and its reversal are opposite numbers. This is established by noticing that $\operatorname{prod}[$ string $]+\operatorname{prod}[$ reversal $]=[$ string $] \times[$ string $]$ (i.e., the result of counting one $l \dot{m}$ term, with the appropriate sign, for each $l$ with each $m$ in the string, including itself). However, for a closed string, [string] $\times$ [string] must be zero; this is because in $L$-closed strings the contribution from each index sums down to zero, given that each index multiplies every other in equal numbers under $\rightarrow$ and $\leftarrow$. Hence this lemma.

Lemma 3.-The cross prod [string] $\times$ [reversal] of a shifted string and its shifted reversal (i.e., the result of counting one $l \dot{m}$ term, with the appropriate sign, for each $l$ in the string and each $m$ in the reversal) is zero. In fact, the cross prod is separately zero for each index in the string with all the indices in its reversal, again because the reversal (as the original string) is $L$-closed and has $\#[\vec{m}]=\#[\overleftarrow{m}]$. Hence this lemma.

Lemma 4.- All self-splicings can be brought into a normal form given by the concatenation (")") of the shifted string and its shifted reversal, for appropriate shifts. This does not change the prod of the self-splicing.

Proof--Hence, prod[self-splicing] is given by

$$
\begin{aligned}
& \operatorname{prod}[\text { shifted string } \mid \text { shifted reversal }]= \\
& \quad \operatorname{prod}[\text { string }]+\operatorname{prod}[\text { reversal }] \\
& +[\text { shifted string }] \times[\text { shifted reversal }]=0,
\end{aligned}
$$

because the first two terms are opposite numbers and the third term vanishes. Hence the proof.

\section{Algebraic expressions for all second-generation TDI observables of length 16}

In this section I give explicit algebraic expressions for all the second-generation TDI observables of length 16, as found in my exhaustive survey, modulus the symmetries discussed in Sec. IIE There is considerable arbitrariness in writing these expressions, corresponding to the selection of representative link strings in each equivalence class, to the convention used in translating strings to sums of $y_{i j}$ measurements, and to the choice of the initial time of evaluation for each observable. Here I list link strings in a normal form whereby each string begins with the largest continuous substring of forward-time indices; I adopt the translation rules given in Sec. IIE [just below Eq. [15)]; and I adjust the time of evaluation to minimize the length of the longest delay sequence in the expression.

X type. The standard 16 -link observable $X_{1}^{16,2}$ is obtained by applying the translation rules to the link string $3^{\prime} 322^{\prime} 22^{\prime} 3^{\prime} 32^{\prime} 233^{\prime} 33^{\prime} 2^{\prime} 2$, and evaluating the resulting expression at the initial time $t_{; 3^{\prime} 322^{\prime} 22^{\prime} 3^{\prime} 3^{\prime}}$ :

$$
\begin{aligned}
& y_{132 ; 322^{\prime} 22^{\prime} 3^{\prime} 3}+y_{231 ; 22^{\prime} 22^{\prime} 3^{\prime} 3}+y_{123 ; 2^{\prime} 22^{\prime} 3^{\prime} 3} \\
+ & y_{321 ; 22^{\prime} 3^{\prime} 3}+y_{123 ; 2^{\prime} 3^{\prime} 3}+y_{321 ; 3^{\prime} 3}+y_{132 ; 3}+y_{231} \\
- & y_{32^{\prime} 1}-y_{123 ; 2^{\prime}}-y_{231 ; 22^{\prime}}-y_{132 ; 322^{\prime}}-y_{231 ; 3^{\prime} 322^{\prime}} \\
- & y_{132 ; 33^{\prime} 322^{\prime}}-y_{321 ; 3^{\prime} 33^{\prime} 322^{\prime}}-y_{123 ; 2^{\prime} 3^{\prime} 33^{\prime} 322^{\prime}}
\end{aligned}
$$

the alternative 16-link observables $X_{1}^{16,4, \pm 1}$ can be writ-

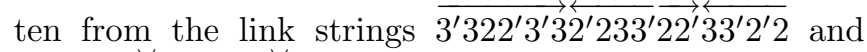

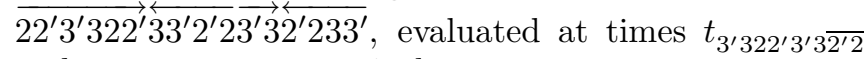
and $t_{22^{\prime} 3^{\prime} 322^{\prime}} \overline{33^{\prime}}$, respectively:

$$
\begin{aligned}
& y_{132 ; 322^{\prime} 3^{\prime} 3 \overline{2^{\prime 2}}}+y_{231 ; 22^{\prime} 3^{\prime} 3 \overline{2^{\prime} 2}}+y_{123 ; 2^{\prime} 3^{\prime} 3 \overline{2^{\prime 2}}} \\
+ & y_{321 ; 3^{\prime} 3 \overline{2^{\prime} 2}}+y_{132 ; 3 \overline{2^{\prime} 2}}+y_{231 ; \overline{2^{\prime} 2}}-y_{321 ; \overline{2^{\prime} 2}}-y_{123 ; \overline{2}} \\
- & y_{231}-y_{132 ; 3}+y_{123 ; \overline{2^{\prime} 3} 3}+y_{321, \overline{2^{\prime} 2} 3^{\prime} 3}-y_{231 ; \overline{2^{\prime} 2} 3^{\prime} 3} \\
- & y_{132 ; 3 \overline{2^{\prime} 2} 3^{\prime} 3}-y_{321 ; 3^{\prime} 3 \overline{2^{\prime} 2} 3^{\prime} 3}-y_{123 ; 2^{\prime} 3^{\prime} 3 \overline{2^{\prime} 2} 3^{\prime} 3}
\end{aligned}
$$

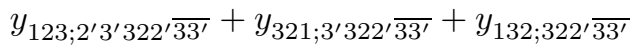

$$
\begin{aligned}
& +y_{231 ; 22^{\prime} \overline{33^{\prime}}}+y_{123 ; 2^{\prime}} \overline{33^{\prime}}+y_{321 ; \overline{33^{\prime}}}-y_{231 ; \overline{33^{\prime}}}-y_{132 ; \overline{3^{\prime}}} \\
& -y_{32^{\prime} 1}-y_{123 ; 2^{\prime}}+y_{132 ; \overline{3^{\prime}} 22^{\prime}}+y_{231 ; \overline{33^{\prime}} 22^{\prime}}-y_{321 ; \overline{33^{\prime}} 22^{\prime}}
\end{aligned}
$$

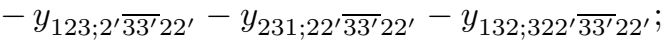

last, the null 16-link observable $X_{1}^{16,4,0}$ can be written

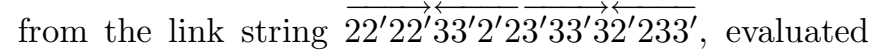
at time $t_{22^{\prime} 22^{\prime}} \overline{33^{\prime} 2^{\prime 2} 2}$ :

$$
\begin{aligned}
& y_{123 ; 2^{\prime} 22^{\prime}} \overline{33^{\prime} 2^{\prime 2} 2}+y_{321 ; 22^{\prime}} \overline{33^{\prime} 2^{\prime 2}}+y_{123 ; 2^{\prime}} \overline{33^{\prime} 2^{\prime} 2}
\end{aligned}
$$

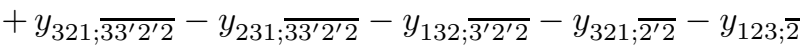

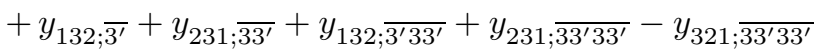

$$
\begin{aligned}
& -y_{123 ; 2^{\prime}} \overline{33^{\prime} 33^{\prime}}-y_{231 ; 22^{\prime}} \overline{33^{\prime} 33^{\prime}}-y_{132 ; 322^{\prime}} \overline{33^{\prime} 33^{\prime}} \text {. }
\end{aligned}
$$

Expressions for the $X$-type observables that use the arms 1,2 and 1,3 are obtained by cyclic index shifts.

U type. The three 16-link $U$-type observables that use the oriented arms $1,1^{\prime}, 2$, and 3 correspond to the link strings

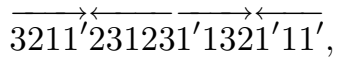

$$
\begin{aligned}
& 3211^{\prime} 231^{\prime} 1321^{\prime} 12311^{\prime}, \\
& \overrightarrow{3211^{\prime} 1321^{\prime} 123} \overleftrightarrow{1^{\prime}} \stackrel{2311^{\prime}}{ } \text {; }
\end{aligned}
$$

applying the translation rules and evaluating at the times 
$t_{3211^{\prime} \overline{2312}}, t_{3211^{\prime} \overline{231^{\prime} 1}}$, and $t_{3211^{\prime} 132 \overline{1}}$, respectively, yields

$$
\begin{aligned}
& y_{231 ; 211^{\prime} \overline{2312}}+y_{123 ; 11^{\prime} \overline{2312}}+y_{312 ; 1^{\prime} \overline{2312}} \\
+ & y_{213 ; \overline{2312}}-y_{123 ; \overline{2312}}-y_{231 ; \overline{312}}-y_{312 ; \overline{12}}-y_{123 ; \overline{2}} \\
- & y_{231}+y_{213 ; \overline{1^{\prime}} 3}+y_{312 ; \overline{11^{\prime}} 3}+y_{231 ; \overline{311^{\prime} 3}}+y_{123 ; \overline{2311^{\prime}} 3} \\
- & y_{213 ; \overline{2311^{\prime}} 3}-y_{312 ; 1^{\prime}} \overline{2311^{\prime} 3}-y_{213 ; 11^{\prime} \overline{2311^{\prime}} 3},
\end{aligned}
$$

$$
\begin{aligned}
& y_{231 ; 211^{\prime} \overline{23} 1^{\prime} 1}+y_{123 ; 11^{\prime} \overline{23} 1^{\prime} 1}+y_{312 ; 1^{\prime} \overline{23} 1^{\prime} 1} \\
+ & y_{213 ; \overline{23} 1^{\prime} 1}-y_{123 ; \overline{23} 1^{\prime} 1}-y_{231 ; \overline{3} 1^{\prime} 1}+y_{213 ; 1}+y_{312} \\
+ & y_{231 ; 3}+y_{123 ; 23}-y_{213 ; \overline{23}}-y_{312 ; 1^{\prime} \overline{23}}-y_{123 ; 11^{\prime} \overline{23}} \\
- & y_{231 ; 211^{\prime}} \overline{23}-y_{312 ; 3211^{\prime} \overline{23}}-y_{213 ; 13211^{\prime} \overline{23}}
\end{aligned}
$$

$$
\begin{aligned}
& y_{231 ; 211^{\prime} 132 \overline{1^{\prime}}}+y_{123 ; 11^{\prime} 132 \overline{1^{\prime}}}+y_{312 ; 1^{\prime} 132 \overline{1^{\prime}}} \\
& +y_{213 ; 132 \overline{1^{\prime}}}+y_{312 ; 32 \overline{1^{\prime}}}+y_{231 ; 2 \overline{1^{\prime}}}+y_{123 ; \overline{1^{\prime}}}-y_{213 ; \overline{1^{\prime}}} \\
& -y_{312}-y_{123 ; 1}-y_{231 ; 21}+y_{213 ; \overline{1^{\prime}} 321}-y_{123 ; \overline{1^{\prime}} 321} \\
& -y_{231 ; 2 \overline{1^{\prime}} 321}-y_{312 ; 32 \overline{1^{\prime}} 321}-y_{213 ; 132 \overline{1^{\prime}} 321} \text {; }
\end{aligned}
$$

the third expression is closest to the $U_{1}$ given in Ref. 11] (but see note 11). Expressions for the $U$-type observables that other sets of oriented arms (i.e., $\left\{2,2^{\prime}, 1,3\right\}$, $\left\{3,3^{\prime}, 1,2\right\},\left\{1,1^{\prime}, 2^{\prime}, 3^{\prime}\right\},\left\{2,2^{\prime}, 1^{\prime}, 3^{\prime}\right\}$, and $\left.\left\{3,3^{\prime}, 1^{\prime}, 2^{\prime}\right\}\right)$ are obtained by cyclic and noncylic index shifts.

E type. The three 16-link E-type observables that use the oriented arms $1,1^{\prime}, 2^{\prime}$, and 3 correspond to the link strings

$$
\begin{aligned}
& \overrightarrow{11^{\prime} 132^{\prime} 1^{\prime} 12^{\prime}} \overleftrightarrow{3} \overrightarrow{1^{\prime} 2^{\prime}} \stackrel{\leftrightarrow}{311^{\prime}} \overrightarrow{3} \overleftarrow{2^{\prime}}
\end{aligned}
$$

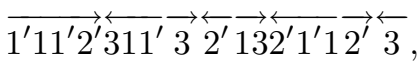

$$
\begin{aligned}
& \overrightarrow{11^{\prime} 2^{\prime}} \overleftrightarrow{3} \overrightarrow{1^{\prime} 132^{\prime} 1^{\prime} 1} \overrightarrow{2^{\prime}} \overleftrightarrow{311^{\prime}} \overrightarrow{3} \overleftarrow{2^{\prime}}
\end{aligned}
$$

applying the translation rules and evaluating at the times $t_{11^{\prime} 13 \overline{2^{\prime} 1^{\prime} 1} 2^{\prime}}, t_{1^{\prime} 11^{\prime} 2^{\prime}} \overline{311^{\prime} 3}$, and $t_{11^{\prime} 2^{\prime} \overline{3} 1^{\prime} 13 \overline{2^{\prime}}}$, respectively, yields:

$$
\begin{aligned}
& y_{312 ; 1^{\prime} 13 \overline{2^{\prime} 1^{\prime} 1} 2^{\prime}}+y_{213 ; 13 \overline{2^{\prime} 1^{\prime} 1} 2^{\prime}}+y_{312 ; 3 \overline{2^{\prime} 1^{\prime} 1} 2^{\prime}} \\
+ & y_{231 ; \overline{2^{\prime} 1^{\prime} 1} 2^{\prime}}-y_{321 ; \overline{2^{\prime} 1^{\prime} 1} 2^{\prime}}-y_{213 ; \overline{1^{\prime} 1} 2^{\prime}}-y_{312 ; \overline{1} 2^{\prime}}+y_{322^{\prime} 1} \\
- & y_{231}+y_{213 ; \overline{1^{\prime}} 3}+y_{321 ; \overline{2^{\prime} 1^{\prime} 3}}-y_{231 ; \overline{2^{\prime} 1^{\prime}} 3}-y_{312 ; 3 \overline{2^{\prime} 1^{\prime} 3}} \\
- & y_{213 ; 13 \overline{2^{\prime} 1^{\prime} 3}}+y_{231 ; \overline{3} 1^{\prime} 13 \overline{2^{\prime} 1^{\prime}} 3}-y_{321 ; \overline{3} 1^{\prime} 13 \overline{2^{\prime} 1^{\prime} 3} 3},
\end{aligned}
$$

$$
\begin{aligned}
& y_{213 ; 11^{\prime} 2^{\prime}} \overline{311^{\prime}} 3+y_{312 ; 1^{\prime} 2^{\prime}} \overline{311^{\prime}} 3+y_{213 ; 2^{\prime}} \overline{311^{\prime} 3} \\
+ & y_{321 ; \overline{311^{\prime}} 3}-y_{231 ; \overline{311^{\prime}} 3}-y_{312 ; \overline{11^{\prime}} 3}-y_{213 ; \overline{1^{\prime}} 3}+y_{231} \\
- & y_{32^{\prime} 1}+y_{312 ; \overline{1} 2^{\prime}}+y_{231 ; \overline{31} 2^{\prime}}-y_{321 ; \overline{31} 2^{\prime}}-y_{213 ; 2^{\prime} \overline{31} 2^{\prime}} \\
- & y_{312 ; 1^{\prime} 2^{\prime} \overline{312^{\prime}}}+y_{321 ; \overline{2}^{\prime}} 11^{\prime} 2^{\prime} \overline{312^{\prime}}
\end{aligned}
$$

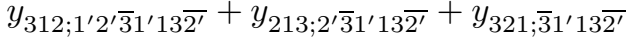

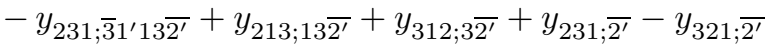

$$
\begin{aligned}
& -y_{21^{\prime} 3}-y_{312 ; 1^{\prime}}+y_{321 ; \overline{2^{\prime}} 11^{\prime}}-y_{231 ; \overline{2^{\prime}} 11^{\prime}}-y_{312 ; 3 \overline{2^{\prime}} 11^{\prime}} \\
& -y_{213 ; 13 \overline{2^{\prime}} 11^{\prime}}+y_{231 ; \overline{3} 1^{\prime} 13 \overline{2^{\prime}} 11^{\prime}}-y_{321 ; \overline{3} 1^{\prime} 13 \overline{2^{\prime}} 11^{\prime}} \text {; }
\end{aligned}
$$

the third expression is closest to the $E_{1}$ given in Ref. [11]. Expressions for the other possible sets of oriented arms (i.e., $\left\{2,2^{\prime}, 1,3^{\prime}\right\}$ and $\left\{3,3^{\prime}, 1^{\prime}, 2\right\}$ ) are obtained by cyclic index shifts.

P type. The three 16 -link $P$-type observables that use the oriented arms $1,1^{\prime}, 2$, and $3^{\prime}$ correspond to the link strings

$$
\begin{aligned}
& \overrightarrow{3^{\prime} 1^{\prime} 11^{\prime}} \stackrel{\leftarrow}{2} \overrightarrow{3^{\prime}} \stackrel{11^{\prime} 3^{\prime}}{21} \overleftrightarrow{3^{\prime}} \overrightarrow{2} \overleftrightarrow{1^{\prime} 12}
\end{aligned}
$$

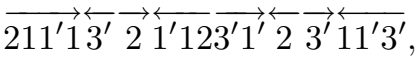

$$
\begin{aligned}
& \overrightarrow{211^{\prime}} \overleftarrow{2} \overrightarrow{3^{\prime} 1^{\prime} 1} \overleftarrow{3^{\prime}} \overrightarrow{2} \overleftrightarrow{1^{\prime} 12} \overrightarrow{3^{\prime}} \overleftrightarrow{11^{\prime} 3^{\prime}} \text {; }
\end{aligned}
$$

applying the translation rules and evaluating at the times $t_{3^{\prime} 1^{\prime} 11^{\prime} \overline{2} 3^{\prime} \overline{11^{\prime}}}, t_{211^{\prime} 1 \overline{3^{\prime}} 2 \overline{1^{\prime} 1}}$, and $t_{211^{\prime} \overline{2} 3^{\prime} 1^{\prime} 1 \overline{3^{\prime}}}$, respectively, yields

$$
\begin{aligned}
& y_{132 ; 1^{\prime} 11^{\prime} \overline{2} 3^{\prime} \overline{11^{\prime}}}+y_{213 ; 11^{\prime} \overline{2} 3^{\prime} \overline{11^{\prime}}}+y_{312 ; 1^{\prime} \overline{2} 3^{\prime} \overline{11^{\prime}}} \\
& +y_{213 ; \overline{2} 3^{\prime} \overline{11^{\prime}}}-y_{123 ; \overline{2} 3^{\prime} \overline{11^{\prime}}}+y_{132 ; \overline{11^{\prime}}}-y_{312 ; \overline{11^{\prime}}}-y_{213 ; \overline{1^{\prime}}} \\
& -y_{13^{\prime} 2}+y_{123 ; \overline{2} 3^{\prime}}+y_{312 ; \overline{12} 3^{\prime}}-y_{132 ; \overline{12} 3^{\prime}}+y_{123 ; \overline{2} 3^{\prime} \overline{12} 3^{\prime}} \\
& -y_{213 ; \overline{2} 3^{\prime} \overline{12} 3^{\prime}}-y_{312 ; 1^{\prime} \overline{2} 3^{\prime} \overline{12} 3^{\prime}}-y_{123 ; 11^{\prime} \overline{2} 3^{\prime} \overline{12} 3^{\prime},}
\end{aligned}
$$

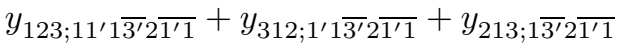

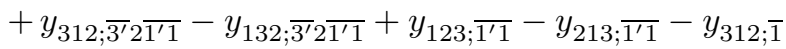

$$
\begin{aligned}
& -y_{123}+y_{132 ; \overline{3^{\prime}} 2}+y_{213 ; \overline{1^{\prime} 3^{\prime}} 2}-y_{123 ; \overline{1^{\prime} 3^{\prime}} 2}+y_{132 ; \overline{3^{\prime}} 2 \overline{1^{\prime} 3^{\prime}} 2} \\
& -y_{312 ; \overline{3^{\prime}} 2 \overline{1^{\prime} 3^{\prime}} 2}-y_{213 ; 1 \overline{3^{\prime}} 2 \overline{1^{\prime} 3^{\prime}} 2}-y_{132 ; 1^{\prime} 1 \overline{3^{\prime}} 2 \overline{1^{\prime} 3^{\prime}} 2} \text {, }
\end{aligned}
$$

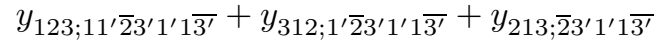

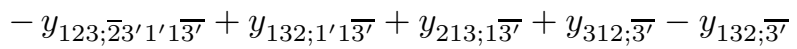

$$
\begin{aligned}
& +y_{123 ; \overline{2}}-y_{213 ; \overline{2}}-y_{312 ; 1^{\prime} \overline{2}}-y_{123 ; 11^{\prime} \overline{2}}+y_{132 ; 3^{\prime} 211^{\prime} \overline{2}} \\
& -y_{312 ; \overline{3^{\prime}} 211^{\prime} \overline{2}}-y_{213 ; 1 \overline{3^{\prime}} 211^{\prime} \overline{2}}-y_{132 ; 1^{\prime} 1 \overline{3^{\prime}} 211^{\prime} \overline{2}} \text {; }
\end{aligned}
$$

the third expression is closest to the $P_{1}$ given in Ref. [11]. Expressions for the other possible sets of oriented arms (i.e., $\left\{2,2^{\prime}, 1^{\prime}, 3\right\}$ and $\left.\left\{3,3^{\prime}, 1,2^{\prime}\right\}\right)$ are obtained by cyclic index shifts. 
[1] LISA Study Team, LISA: Laser Interferometer Space Antenna for the detection and observation of gravitational waves, Pre-Phase A Report, 2nd ed. (Max Planck Institut für Quantenoptik, Garching, Germany, 1998).

[2] LIGO: A. Abramovici et al., Science 256, 325 (1992); VIRGO: B. Caron et al., Class. Quantum Grav. 14, 1461 (1997); GEO: H. Lück et al., ibid., 1471 (1997); TAMA: M. Ando et al., Phys. Rev. Lett. 86, 3950 (2001).

[3] See, e.g., S. A. Hughes, Ann. Phys. 303, 142 (2003), and references therein.

[4] M. Tinto and J. W. Armstrong, Phys. Rev. D 59, 102003 (1999).

[5] J. W. Armstrong, F. B. Estabrook, and M. Tinto, Astrophys. J. 527, 814 (1999); J. W. Armstrong, F. B. Estabrook, and M. Tinto, Class. Quant. Grav. 18, 4059 (2001); see also G. Giampieri et al., Opt. Comm. 123, 669 (1996) for a frequency-domain laser-noise subtraction scheme related to TDI.

[6] F. B. Estabrook, M. Tinto, and J. W. Armstrong, Phys. Rev. D 62, 042002 (2000).

[7] S. V. Dhurandhar, K. R. Nayak, and J.-Y. Vinet, Phys. Rev. D 65, 102002 (2002); K. R. Nayak and J.-Y. Vinet, ibid., 70, 102003 (2004).

[8] D. A. Shaddock, Phys. Rev. D 69, 022001 (2004).

[9] N. Cornish and R. W. Hellings, Class. Quant. Grav. 20, 4851 (2003).

[10] D. A. Shaddock, M. Tinto, F. B. Estabrook, and J. W. Armstrong, Phys. Rev. D 68, 061303(R) (2003).

[11] M. Tinto, F. B. Estabrook, and J. W. Armstrong, Phys. Rev. D 69, 082001 (2004).

[12] M. Tinto, D. A. Shaddock, J. Sylvestre, and J. W. Armstrong, Phys Rev. D 67, 122003 (2003).

[13] T. A. Prince, M. Tinto, S. L. Larson, and J. W. Arm- strong, Phys. Rev. D 66, 122002 (2002).

[14] D. Summers, "Algorithm tradeoffs," oral presentation, 3rd progress meeting of the ESA-funded LISA PMS Project, ESTEC, the Netherlands, February 2003; D. Summers and D. Hoyland, Class. Quantum Grav. 22, S249 (2005).

[15] See, e.g., G. M. Jenkins and D. G. Watts, (Holden-Day, San Francisco, 1968).

[16] C. W. Misner, K. S. Thorne, and J. A. Wheeler, Gravitation (W. H. Freeman, San Francisco, 1973).

[17] J. W. Armstrong, F. B. Estabrook, and H. D. Wahlquist, Astrophys. J. 318, 536 (1987); B. Bertotti et al., Astron. Astrophys. 296, 13 (1995); M. Tinto, Class. Quant. Grav. 19, 1767 (2002); J. W. Armstrong, L. Iess, P. Tortora, and B. Bertotti, Astrophys. J. 599, 806 (2003).

[18] A. N. Lommen and D. C. Backer, Astrophys. J. 562, 297 (2001); F. A. Jenet, A. Lommen, S. L. Larson, and L. Wen, Astrophys. J. 606, 799 (2004).

[19] F. B. Estabrook and H. D. Wahlquist, Gen. Rel. Grav. 6, 439 (1975); H. D. Wahlquist, ibid., 19, 1101 (1987).

[20] M. Vallisneri, Phys. Rev. D 71, 022001 (2005); see also www.vallis.org/syntheticlisa

[21] N. Ashby, Living Rev. Relativity 6, 1 (2003). www.livingreviews.org/lrr-2003-1

[22] M. Tinto, J. W. Armstrong, and F. B. Estabrook, Phys Rev. D 63, 021101(R) (2000); C. J. Hogan and P. L. Bender, ibid., 64, 062002 (2001).

[23] M. Tinto, M. Vallisneri, and J. W. Armstrong, Phys. Rev. D 71, 041101(R) (2005).

[24] D. A. Shaddock, B. Ware, R. E. Spero, and M. Vallisneri, Phys. Rev. D 70, 081101(R) (2004). 